\title{
Performance evaluation of macro-assisted small cell energy savings schemes
}

\author{
Emmanuel Ternon ${ }^{1}$, Patrick Kwadwo Agyapong $^{1}$ and Armin Dekorsy ${ }^{2}$
}

\begin{abstract}
Sleep mode techniques for small cells provide an effective means to mitigate excessive energy consumption and interference associated with ultra-dense small cell deployments. Nevertheless, such techniques can impact network performance in traditional deployments. In this paper, we show that in the particular case of macro-controlled small cells, such as in the Phantom Cell Concept (PCC) architecture, effective macro-assisted energy savings schemes can be employed to reduce the network energy consumption at practically no additional cost to the network and no hit to user quality of service (QoS). We introduce a heuristic algorithm suitable for real network operation, that minimizes the energy consumption in the small cell network by selecting the small cell offering the best signal-to-interference-plusnoise ratio (SINR). We apply this heuristic to three energy savings schemes realizable in the PCC architecture. These schemes,based on the macro-assisted user equipment (UE)-small cell connection establishment paradigm, are a downlink (DL) signalling based scheme, an uplink (UL) signalling based scheme and a database-aided scheme. We derive a power consumption model for a representative PCC small cell, which allows to quantify the percentage of energy consumed by a small cell in sleep mode with respect to a fully operational small cell for the three energy savings schemes considered. Furthermore, we characterize the connection latency associated with each scheme and evaluate the impact on system performance. System-level simulations show that, even with the associated connection delays, the macro-assisted energy-saving schemes can yield energy savings of more than $45 \%$ while at the same time introduce throughput gains of up to $25 \%$.
\end{abstract}

Keywords: Heterogeneous networks; Energy savings; Phantom cell; Small cell sleep mode; Small cell power model; Connection latencies

\section{Introduction}

Ultra-dense small cell deployments are seen as a necessary means to address the anticipated thousandfold increase in mobile network traffic by 2020 [1, 2]. However, deploying a large number of small cells poses several challenges such as increased network complexity and management, unplanned interference and potentially increased energy consumption (and thus operational costs).

Decoupling coverage and capacity provisioning could make it easier to deploy and manage a large number of small cells. The Phantom Cell Concept (PCC) is a system architecture taking this approach. In the $\mathrm{PCC}$, the control plane (C-plane) and the (U-plane) are separated, with

\footnotetext{
*Correspondence: ternon@docomolab-euro.com

1 DOCOMO Communications Laboratories Europe GmbH, Landsberger Strasse 312, 80687 Munich, Germany

Full list of author information is available at the end of the article
}

a macro cell layer providing ubiquitous $\mathrm{C}$-plane coverage and a small cell layer providing U-plane capacity [3].

Operating a large number of small cells can also be expensive from a user quality of service (QoS) point of view, due to high interference levels, and from the point of view of operators due to increased operating expenses as a result of increased energy consumption. Sleep mode techniques, where a small cell is in a low power stand-by mode with limited operational capabilities, can help to alleviate such issues. However, putting small cells to sleep could potentially impact the performance of the network. First, it takes a non-negligible amount of time to wake up sleeping small cells and make them fully operational. Thus, a network with such capability may be slow to react to any sudden increase in capacity demand.

Secondly, employing sleep mode techniques could result in a sub-optimal link establishment and consequent undesirable interference environment and user experience.

\section{它 Springer}

C 2015 Ternon et al. Open Access This article is distributed under the terms of the Creative Commons Attribution 4.0 International License (http://creativecommons.org/licenses/by/4.0/), which permits unrestricted use, distribution, and reproduction in any medium, provided you give appropriate credit to the original author(s) and the source, provide a link to the Creative Commons license, and indicate if changes were made. 
This is because it is difficult for a user equipment (UE) to discover a nearby sleeping small cell $[4,5]$. As a result of such discovery issues, UEs may connect to active small cells which are overloaded or offer a poor link quality, leading to suboptimal throughput rates. Finally, employing sleep mode techniques could lead to a longer connection setup time for a UE connecting to a discovered sleeping cell, which could impact the user quality of experience [6].

In the particular case of macro-controlled small cells, such as in the PCC architecture, effective macro-assisted energy savings schemes based on sleep mode techniques which overcome the above-mentioned issues can be employed at practically no additional cost to the network. These energy savings schemes can be implemented in real-world small cell network deployments with minimal additional signalling with the help of a heuristic algorithm that we introduce in this paper. This heuristic algorithm minimizes the energy consumption of the network by selecting for the small cell offering the best signal-tointerference-plus-noise ratio (SINR) to a connecting UE, provided this small cell can meet the data rate requirement of this UE.

We adapt two energy savings schemes based on sleep mode techniques, namely a downlink (DL) signalling-based scheme and an uplink (UL) signallingbased scheme [6], originally proposed for traditional heterogeneous networks (HetNets), to the PCC architecture, to demonstrate how macro-assisted UE-small cell connection establishment procedures can be used to realize energy savings without impacting user QoS. A third energy savings scheme natively enabled by the PCC architecture, the database-aided scheme, is also presented. This scheme has been proposed by the authors in $[4,5]$ and aims at combining the advantages of the two schemes above to realize more energy savings. We apply the heuristic algorithm introduced in this paper to quantify the achieved energy savings and user QoS realizable with these three energy savings schemes.

In order to realistically quantify the energy savings potential, a power consumption model of a representative PCC small cell base station (BS) is derived for the schemes studied. Furthermore, a detailed characterization of connection setup latency for all the studied schemes is also presented to properly quantify the impact of realistic implementation conditions on user QoS of the considered energy savings schemes. System-level simulations implementing the three considered energy savings schemes using the introduced heuristic algorithm show that energy savings of more than $45 \%$ can be achieved, very close to the $52 \%$ energy savings achievable with state-of-the-art optimization algorithms. However, unlike the state-ofthe-art algorithm in which UE throughput is sometimes severely degraded, the heuristic algorithm introduced in this paper yields throughput gains of up to $25 \%$ with respect to a baseline scheme not implementing energy savings.

This paper is organized as follows. First of all, an overview of state-of-the-art techniques for energy savings in HetNets small cell deployments, based on sleep mode techniques for small cells, is laid out in Section 2 as a background for the work presented in this paper. Then, the system model considered, based on the PCC architecture, is introduced in Section 3. In Section 4, we formulate the energy savings problem mathematically as a constrained minimization problem, and introduce a heuristic algorithm to solve it with minimal impact on network signalling. Details of the schemes employed to realize energy savings are also presented. Subsequently, in Section 5, we provide a power consumption model for a representative PCC small cell BS in sleep mode, and determine the signalling latencies generated by the UE-small cell connection establishment procedure for each of the considered energy savings scheme in Section 6. Finally, system-level simulation performance results, which show the benefits brought by the energy savings schemes, are introduced and analysed in Section 7.

\section{Background}

Energy consumption in wireless networks, especially on the air interface, is well-studied in the literature. Given the increasing awareness of the detrimental ecological and financial impact of rising energy consumption, both governments and the industry are also investing heavily in research activities to realize green networks. For example, the EU-funded EARTH project [7] laid the foundations for modelling energy consumption in the radio access network and provided new techniques and key insights on how the network could be deployed and managed to reduce energy consumption. The $5 \mathrm{GrEEn}$ project [8] is also studying clean slate approaches to design environmentally friendly future mobile networks. Standard bodies such as European Telecommunications Standards Institute (ETSI), through the ETSI Environmental Engineering (ETSI EE) committee [9] are also investigating and providing guidelines towards the environmentally friendly design of components for telecommunication networks.

Due to the multiple components present in a wireless network, energy savings schemes can be implemented at different levels, such as the core network $(\mathrm{CN})$ or the radio access network (RAN). Approaches such as softwaredefined networking and network functions virtualization could provide some energy savings in the $\mathrm{CN}$ via the ability to support dynamic scale-in/scale-out of network capabilities on demand [2]. Nevertheless, the RAN still consumes about $80 \%$ of the energy in cellular networks [10]. Thus, a lot of research activity has focused on techniques to reduce BS energy consumption in particular. 
These cover techniques to reduce energy consumption on the air interface-either based on power control [11, 12], discontinuous transmission (DTX) [13] or both [14]; techniques to design more energy-efficient BS components $[15,16]$, and techniques to manage overall BS energy consumption through switching on/off some components depending on the prevailing network load $[17,18]$.

In large BSs (e.g., macro cell BSs) where the power amplifier takes a significant proportion of the overall energy consumed, techniques to manage air interface energy consumption could be very efficient, providing up to $40 \%$ energy savings [19]. However, such techniques do not provide much energy savings gain in small cell BSs, where most of the energy is consumed in the baseband and radio frequency (RF) parts of the circuitry [20]. For such BSs, sleep mode techniques, where a large number of components in the BS circuitry are turned off, provide a more efficient means to realize energy savings by exploiting the variability in user and traffic distribution across the network. This is particularly true for densely deployed HetNets, where a large number of small cells are deployed in a certain area to meet the capacity requirements of peak traffic hours, while only a fraction of these small cells are needed in low traffic hours [21]. A good overview of sleep mode techniques for HetNets is provided in [22] and [23]. Several issues that arise with the implementation of sleep mode techniques, particularly with small cell discovery and UE-small cell channel measurement, are well documented in the literature (see e.g., $[24,25]$ ) and also briefly highlighted in Section 1.

Two energy savings schemes based on sleep mode techniques have been proposed by the 3rd Generation Partnership Project (3GPP) to overcome the aforementioned issues, namely a DL signalling based scheme and an UL signalling based scheme [6]. In the DL signalling-based scheme, small cells in sleep mode still send beacon signals to remain discoverable to UEs. In the UL signalling based scheme, however, the UEs transmit wake-up signals to alert sleeping small cells of their presence, so that the small cells can wake up from the sleep mode. The small cells do not transmit any signal when they are in sleep mode in the UL signalling based scheme. Both approaches have been developed for traditional HetNets where macro cells and small cells operate independently. In this paper, we adapt both schemes to the PCC architecture, to take advantage of optimization opportunities available with a macro-assisted UE-small cell connection paradigm made possible with this architecture. In addition, we introduce an additional scheme, the database-aided energy savings scheme, which aims at overcoming the drawbacks of both the UL signalling based scheme and the DL signalling based scheme while realizing all their advantages.

Several models and metrics are available in the literature to characterize and quantify potential energy savings in the network. For instance both the ETSI EE [26] and the EARTH Project [27] introduce metrics to quantify network energy consumption. The EARTH Project also introduces an additive component-based model to characterize the overall energy consumption of a BS, applicable to four BS form factors [20]. Similar component-based models have also been proposed in the literature [22, 23]. In this paper, we define a typical small cell BS form factor expected to be used in the PCC architecture and employ the EARTH power model to characterize the sleep mode energy consumption of such a BS for the three energy savings schemes identified earlier.

Energy savings schemes based on sleep mode techniques can further introduce connection latencies to the UE-small cell connection procedure, which may have an impact on the achievable energy savings and QoS delivered to users. Several works, such as [28] or [29], provide some analysis of potential network delays in connection setup introduced by sleep mode techniques, but this evaluation is not fully applicable in architectures with Cplane/U-plane split and centralized control of small cells by a macro BS. In addition, these studies do not quantify the impact of these delays on potential energy savings and user QoS. In [4] and [5], the authors evaluate energy savings for a macro-assisted database-aided energy savings scheme for the PCC under different small cell and user deployment scenarios. However, these studies do not consider the impact of signalling latencies in the UE-small cell connection establishment procedure. A mathematical model to study the relationship between achievable energy savings by sleep mode BSs and the impact of delays on user QoS is provided in [30], but this analysis assumes independent operation of small cells and macro cells. In this paper, we thoroughly characterize the UE-small cell connection latencies present in the energy savings schemes based on sleep mode techniques considered in this paper, using typical time values for the various signalling procedures, and evaluate their impact on system performance.

This paper adds to the literature in three key areas. First of all, it adapts two energy savings schemes, namely the DL signalling based scheme and the UL signalling based scheme to the PCC HetNet architecture, where a master-slave relationship exists between the macro cell and small cells. It also introduces a novel database-aided energy savings scheme, which combines the advantages of both the UL and DL signalling-based schemes while mitigating their drawbacks. Secondly, it defines a typical small cell BS in the PCC architecture and derives a power model to characterize small cell BS energy consumption for the different energy savings schemes. Finally, it characterizes the connection latencies introduced by the different energy savings schemes and quantifies the impact of these connection delays on the realizable energy 
savings and user throughput. The next section introduces the system model considered.

\section{System model}

\subsection{System architecture}

We consider the PCC architecture [3] as illustrated in Fig. 1. Systems deployed according to the PCC architecture are comprised of two overlaid networks:

- A macro network, where several macro cell BSs are deployed to provide coverage. Macro cells operate in lower frequency bands (e.g., $2 \mathrm{GHz}$ ), using legacy standards, such as 3GPP Long Term Evolution (LTE), guaranteeing backwards compatibility for legacy UEs, i.e., UEs which only support legacy standards;

- A small cell network, where a large number of small cells are deployed to provide localized throughput and capacity. Small cells operate in higher frequency bands (e.g., $3.5 \mathrm{GHz}$ ), and are each connected to a macro cell through a backhaul link. Unlike conventional small cells in traditional HetNets, PCC small cells do not transmit cell-specific signals, such as primary synchronization signal (PSS)/secondary synchronization signal (SSS), cell-specific reference signal (CRS), or master information block (MIB)/system information block (SIB). Instead, they are assumed to transmit a new type of pilot symbols, discovery reference signals (DRS), required for small cell discovery and initial channel estimation purposes. The design of such signals is currently being studied (see e.g., [31, 32]). A key design challenge for such pilot symbols is the trade-off between discovery and channel measurement accuracy, and energy consumption $[3,33]$. In this paper, we consider that 5 $\%$ of the radio frame is occupied by DRS pilots symbols used for the purpose of small cell discovery and channel estimation. This provides a good trade-off between discovery/channel measurement reliability and energy consumption [33].

PCC systems enable to separate the serving points of $\mathrm{C}$-plane and U-plane, with macro cells responsible for $\mathrm{C}$-plane connectivity and small cells handling U-plane connectivity. As a result, a UE can have dual connectivity to a macro cell (for coverage) and a small cell (for capacity) [3]. We consider a configuration where a small cell is directly connected to the $\mathrm{CN}$ and takes care of all the baseband processing of data locally, only exchanging lowthroughput signalling information with the macro cell via the backhaul link [3]. Multiple U-plane connectivity-using bearer split, where user data from a single Internet Protocol (IP) flow is both transmitted from the macro cell and the small cell, is not considered within the scope of this paper, due to required additional complexity (e.g., scheme to properly re-order packets coming from two separate data streams) [24].

Also, not considering bearer split places less stringent requirements on the backhaul between the small cell BSs. This is because the backhaul is only needed to exchange control information, rather than high-throughput user data. However, in more advanced configurations (e.g., with bearer split), the backhaul requirements become more stringent.

\subsection{UE connectivity modes}

Two UE connectivity modes are considered for the system, namely a single connectivity mode where both $\mathrm{C}$-plane band U-plane connections to a UE are handled by a macro cell, and a dual connectivity mode where a UE's C-plane connection is handled by a macro cell and its U-plane

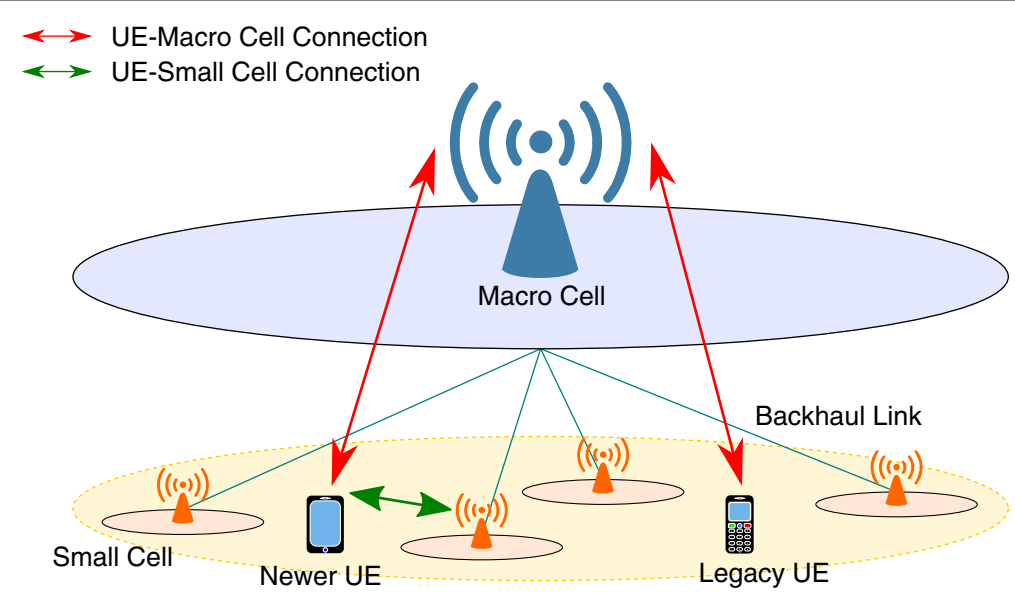

Fig. 1 Representation of the PCC network architecture 
connection by a small cell. While newer UEs support both connection modes, legacy UEs only support the single connectivity mode.

Single connectivity to a small cell is difficult to implement since PCC small cells do not transmit cell-specific signals, which makes it challenging for UEs to connect to small cells in a stand-alone fashion [3]. Furthermore, this scenario would lead to significant network signalling overhead and a higher rate of link failures, due to frequent handover procedures necessary to provide seamless mobility for relatively fast-moving UEs. As a result, we do not consider the case of single connectivity to a small cell within the scope of this paper.

In the considered system concept, UEs which connect to small cells are required to maintain a connection to a macro cell so as to enable the implementation of macro-assisted UE small cell connection establishment mechanisms, introduced in Section 3.4. This implies that the connection to a macro cell is a pre-requisite for the connection to a small cell. Note that this configuration corresponds to the first scenario described in Section 6.1.1 of [34], in which a UE is in coverage of both a macro cell and a small cell simultaneously.

\subsection{Small cell states}

We consider three small cell states, namely on, off, and sleep, as illustrated in Fig. 2. In the rest of the paper, the terms state and mode are used interchangeably. In the on state, small cells are fully operational and consume the highest amount of energy. In the sleep state, small cells are in a stand-by mode where they cannot serve any user, but consume a reduced amount of energy compared to a small cell in the on state. The energy consumed in the sleep state is, however, non-negligible, since the small cell is required to be activated back to the on state very quickly [35]. In the off state, a small cell is completely deactivated, with almost all of its circuitry components switched off. In the

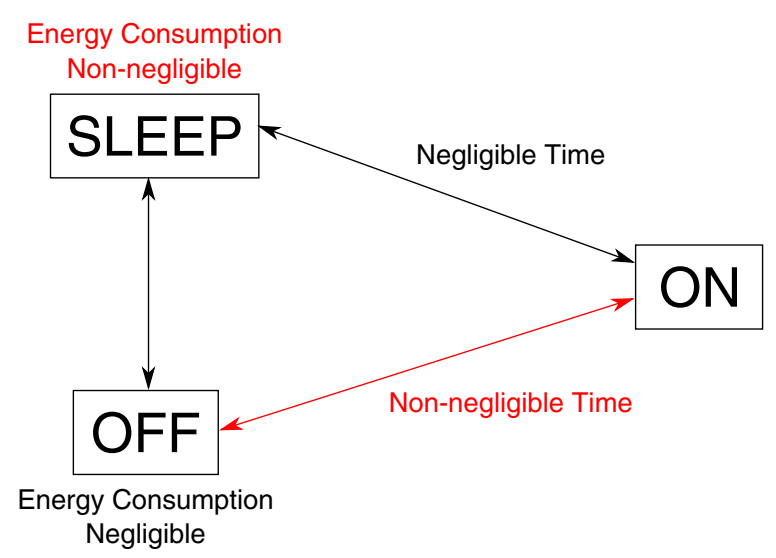

Fig. 2 Overview of the small cell states and their transitions off state, the small cell consumes a negligible amount of energy compared to the on or sleep states, but with current technology, it takes a non negligible amount of time to transition from the off to the on state. This high transition time between off and on state may prevent the network to deliver the required QoS to UE in case of sudden traffic increases. Thus, schemes that implement such transitions are not suitable for scenarios where traffic and user positions dynamically vary and cannot be known in advance. Our goal is to support such scenarios in normal network operation. As a result, we only focus on energy savings schemes based on transitions between small cell on state and sleep state, and do not consider the off state in the rest of this paper.

\subsection{Macro-assisted small cell connection establishment}

In traditional HetNet small cell deployments, the connection establishment between a UE and a small cell, as observed in Fig. 3, is independently managed by the UE and the small cell. The UE autonomously selects the small cell to connect to based on e.g., estimations of the UE small cell channels obtained with the help of pilot symbols (e.g., CRS), and subsequently performs the random access (RA) procedure with this small cell. The macro BS is normally not involved in this connection procedure. This stand-alone connection establishment could lead to sub-optimal outcomes where some small cells are heavily loaded whereas others are lightly loaded.

In architectures such as the PCC, where it is assumed that a UE always keeps its connection with a macro cell BS, it is feasible to explore macro-assisted UE small cell connection establishment procedures, as depicted in Fig. 4. Differences between the traditional stand-alone scheme and the macro-assisted scheme are highlighted in red. In contrast to traditional HetNet where the UE makes the decision on which cell to connect to, in macro-assisted schemes, the macro cell, based on information received from several sources, decides which small cell a UE shall make a connection to. This information can be, for instance, UE small cell channel measurements reported from UEs, or the load of each small cell connected to the macro cell reported via the backhaul link. Since most of this information is anyway exchanged during normal system operation, the implementation of macro-assisted schemes is not expected to introduce additional signalling load either on the air interface or on the backhaul. Furthermore, this centralized approach gives possibilities to the macro cell to perform load balancing operations, depending, e.g., on the QoS requirements of each served user. The macro cell can for example trigger a connection between a user and a small cell when a large data packet needs to be delivered for this user.

The next section formulates the energy savings problem in the PCC mathematically and introduces a heuristic 


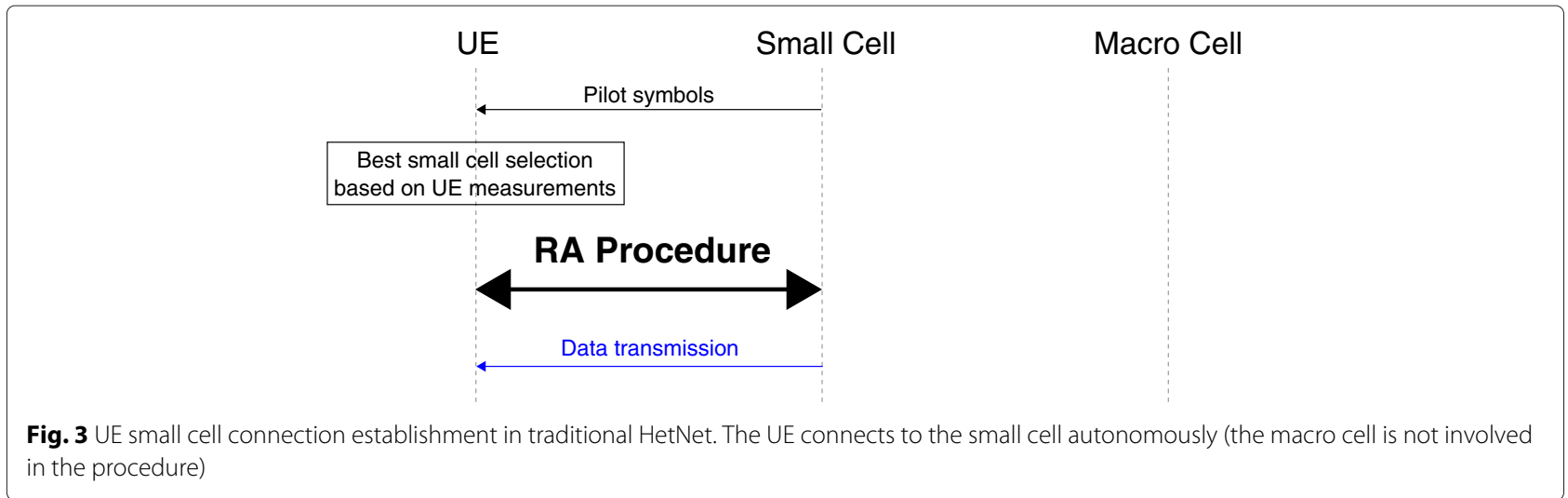

algorithm to solve it. Small cell energy savings schemes based on macro-assisted small cell connection procedures are also introduced.

\section{Small cell energy savings schemes}

The PCC architecture, together with dual-connectivity capability at the UE side, enables us to exploit macroassisted UE-small cell connection establishment procedures to realize energy savings through small cell sleep modes, without impacting the user QoS. In traditional HetNet architectures, small cell sleep mode schemes where the transmitting chain of the small cell is completely switched off are difficult to realize, mostly due to discovery issues of the small cells in sleep mode. Indeed, if a small cell in sleep mode stops transmitting pilot symbols, UEs can neither be aware of its existence, nor obtain an estimation of the UE-small cell channels [4, 5]. Macro- assisted small cell connection establishment procedures overcome this discovery issue, since connections of UEs to small cells are systematically handled by the macro BS, which is always aware of the status of small cells connected to it via the backhaul link. The PCC architecture intrinsically supports the implementation of small cell energy savings schemes which exploit macro-assisted small cell connection procedures.

In this paper, we propose a heuristic to realize energy savings and use it to investigate the extent to which energy savings can be realized in the PCC architecture, taking into account realistic network deployment and operating conditions, as well as realistic implementation considerations for three energy savings schemes. These are the UL signalling-based scheme, the DL signalling-based scheme and the database-aided scheme. The next section introduces the mathematical framing of the energy savings

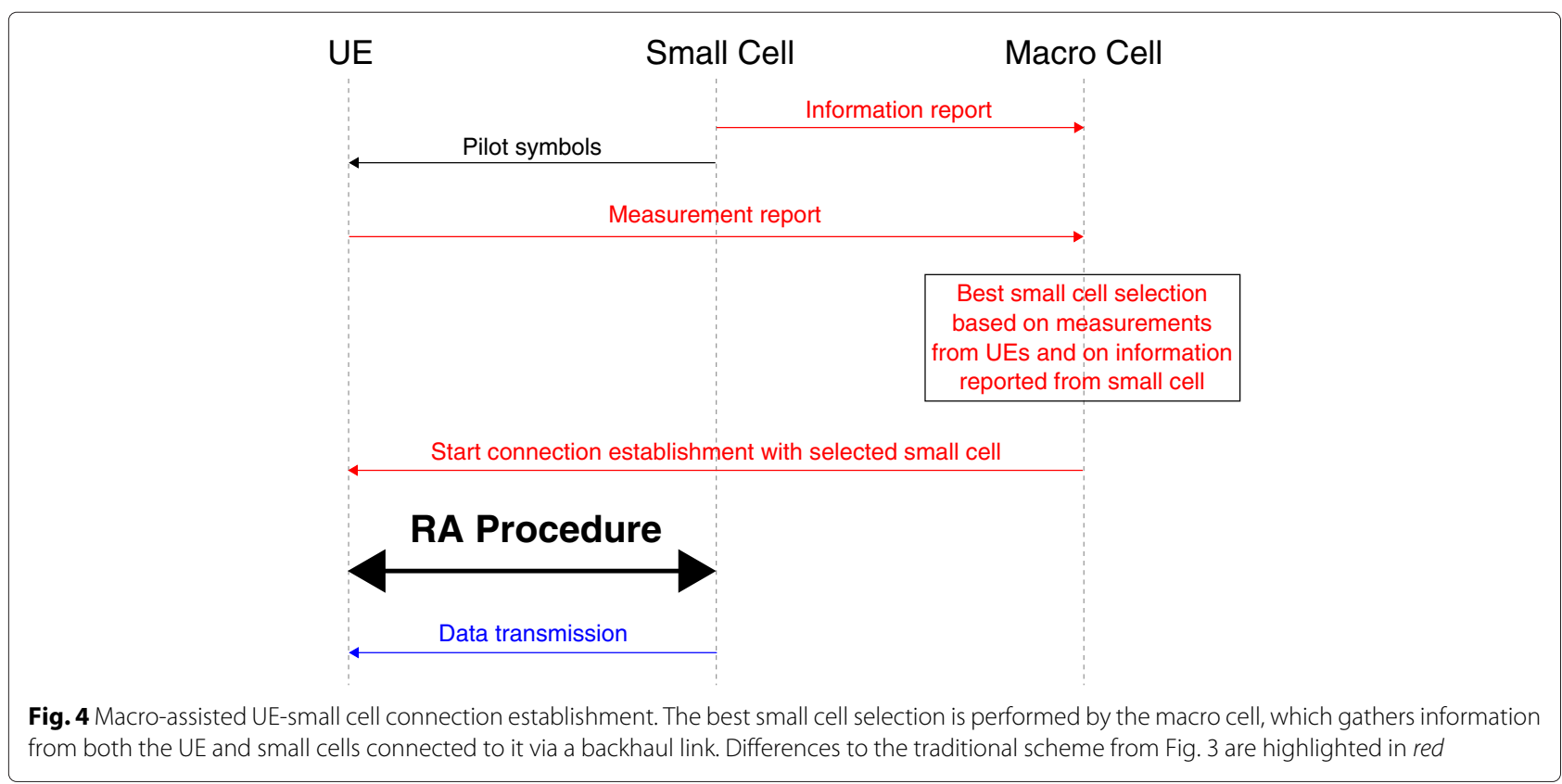


problem and the proposed heuristic algorithm to solve it. This is followed by a detailed description of the energy savings schemes considered.

\subsection{Framing of the energy savings problem}

The goal of energy savings schemes in PCC systems is to minimize the energy consumption of small cells in the network while guaranteeing that the QoS requirements of each user are fulfilled. Mathematically, this optimization problem can be formulated for a given time granularity (e.g., frame, timeslot, several timeslots, hours, etc.) as:

$$
\min _{S_{n}} \sum_{n=1}^{N}\left[S_{n} E_{\text {on }}+\left(1-S_{n}\right) E_{\text {sleep }}\right]
$$

subject to:

$$
\begin{aligned}
& R_{k} \geq \rho_{k}, \quad \forall k \in\{1, \ldots, K\} \\
& S_{n} \in\{0,1\} \\
& E_{\text {on }}>0 \\
& E_{\text {sleep }} \geq 0
\end{aligned}
$$

where $N$ is the total number of small cells in the system, $K$ the number of users in the system, $E_{\text {on }}$ the energy consumed by a small cell in the on state, $E_{\text {sleep }}$ the energy consumed by a small cell in the sleep state, $R_{k}$ the throughput (or data rate) obtained by UE $k, \rho_{k}$ the data rate requirement of user $k$,

$$
\text { and } S_{n}=\left\{\begin{array}{l}
1 \text { if small cell } n \text { is in the on state } \\
0 \text { if small cell } n \text { is in the sleep state }
\end{array}\right.
$$

The data rate metric is used as a proxy to represent the QoS obtained by users. The expression

$$
\sum_{n=1}^{N}\left[S_{n} E_{\text {on }}+\left(1-S_{n}\right) E_{\text {sleep }}\right]
$$

can be rewritten as:

$$
N E_{\text {sleep }}+E_{\text {surplus }} \sum_{n=1}^{N} S_{n}
$$

where $E_{\text {surplus }}=E_{\text {on }}-E_{\text {sleep }}$. Minimizing (6) with respect to $S_{n}$ is therefore equivalent to:

$$
\min _{S_{n}} \sum_{n=1}^{N} S_{n}
$$

Additionally, constraint (2), imposing that the data rate requirement of each UE in the system be fulfilled, is equivalent to a constraint that each BS in the system need to have enough bandwidth to fulfil the data rate requirement of all the UEs it is serving. It is therefore possible to rewrite constraint (2) as:

$$
\begin{aligned}
& \sum_{k=1}^{K} \frac{\rho_{k}}{\omega_{n, k}} \alpha_{n, k} \leq B_{\text {total }, n}, \quad \forall n \in\{1, \ldots, N\}, \\
& \sum_{k=1}^{K} \frac{\rho_{k}}{\omega_{\text {macro }, k}}\left(1-\sum_{n=1}^{N} \alpha_{n, k}\right) \leq B_{\text {total,macro }}, \\
& \sum_{n=1}^{N} \alpha_{n, k} \leq 1, \quad \forall k \in\{1, \ldots, K\} \\
& \alpha_{n, k} \in\{0,1\}, \quad \forall n \in\{1, \ldots, N\}, k \in\{1, \ldots, K\},
\end{aligned}
$$

where $\omega_{n, k}$ (respectively $\omega_{\text {macro,k }}$ ) is the spectral efficiency of the link between small cell $n$ (respectively the macro cell) and user $k$, in bit $/ \mathrm{s} / \mathrm{Hz}, B_{\text {total }, n}$ (respectively $\left.B_{\text {total,macro }}\right)$ the total bandwidth available on small cell $n$ (respectively the macro cell), in $\mathrm{Hz}$,

$$
\text { and } \alpha_{n, k}=\left\{\begin{array}{l}
1 \text { if small cell } n \text { is serving user } k \\
0 \text { if small cell } n \text { is not serving user } k
\end{array}\right.
$$

Ideally, a small cell should be in sleep mode whenever it is not serving any user, but in the on state when serving at least one user. Mathematically, this can be expressed as:

$$
S_{n}=\left\{\begin{array}{l}
1 \text { if } \sum_{k=1}^{K} \alpha_{n, k} \geq 1 \\
0 \text { if } \sum_{k=1}^{K} \alpha_{n, k}=0
\end{array} \quad \forall n \in\{1, \ldots, N\} .\right.
$$

When taking all small cells $N$ into consideration, (13) can be expressed as:

$$
\sum_{n=1}^{N} S_{n}=\left\|\mathbf{A} \mathbf{1}_{K}\right\|_{0}
$$

where $\mathbf{A}:=\left(\alpha_{n, k}\right)_{n, k}, \mathbf{1}_{K}$ is the unity vector of size $K$ and $\|\cdot\|_{0}$ is the $l_{0}$ norm, e.g., the number of non-zero elements of the considered vector. The minimization problem of (1) can then be rewritten as:

$$
\min _{\alpha_{n, k}}\left\|\mathbf{A} \mathbf{1}_{K}\right\|_{0}
$$

subject to:

$$
\begin{aligned}
& \sum_{k=1}^{K} \frac{\rho_{k}}{\omega_{n, k}} \alpha_{n, k} \leq B_{\text {total }, n}, \quad \forall n \in\{1, \ldots, N\}, \\
& \sum_{k=1}^{K} \frac{\rho_{k}}{\omega_{\text {macro }, k}}\left(1-\sum_{n=1}^{N} \alpha_{n, k}\right) \leq B_{\text {total,macro }}, \\
& \sum_{n=1}^{N} \alpha_{n, k} \leq 1, \quad \forall k \in\{1, \ldots, K\} \\
& \alpha_{n, k} \in\{0,1\}, \quad \forall n \in\{1, \ldots, N\}, k \in\{1, \ldots, K\} .
\end{aligned}
$$

Solutions of the above problem take the form of $N \times K$ matrices, where a row with a non-zero sum indicates a 
small cell that should be switched on and the non-zero elements in each such row represent the users that should be served by this small cell. It has been shown in [36] and [37] that problems of this form are intractable for large numbers of small cells and UEs, i.g., the existence of a unique optimal solution is not guaranteed. Nevertheless, good feasible solutions can be found by relaxing the minimization problem and applying the majorization-minimization (MM)algorithm (see [36] for details).

The solution of the problem expressed in (15) to (19) is dependent on the number of users $K$ actively receiving data from the network. This number is fixed when a full buffer traffic model is considered, but potentially frequently varying when a non-full buffer traffic model is considered. In that case, the optimal solution, if it exists, of UE-small cell connections $\alpha_{n, k}$ may frequently vary, which means that UEs would potentially have to connect to a new BS every time a new user starts or finishes receiving data, to guarantee that the energy consumption of the small cell network is always minimized. Due to the significant computation burden and excessive strain on network signalling which would be required to hand over UEs to new small cells, such an approach is impractical for actual network operation.

To overcome the above problems, we propose another approach adapted to non-full buffer traffic assumptions and realizable with minimal additional signalling to perform UE small cell connections. The energy consumed by a given small cell $n$ over a period $\Delta t_{n}$ can be expressed as:

$$
E_{\Delta t_{n}}=P_{\mathrm{on}} t_{\mathrm{on}, n}+P_{\text {sleep }} t_{\mathrm{sleep}, n}
$$

where $P_{\text {on }}$ is the power consumption of a small cell in on mode, in W, $P_{\text {sleep }}$ the power consumption of a small cell in sleep mode, in W, $t_{\mathrm{on}, n}$ the time small cell $n$ is in on mode, in s, and $t_{\text {sleep }, n}$ the time small cell $n$ is in sleep mode, in s. Since a small cell is always either in on mode or in sleep mode, we have $\Delta t_{n}=t_{\mathrm{on}, n}+t_{\mathrm{sleep}, n}$. Therefore, we have:

$$
E_{\Delta t_{n}}=\left(P_{\text {on }}-P_{\text {sleep }}\right) t_{\text {on }, n}+P_{\text {sleep }} \Delta t_{n}
$$

since the term $P_{\text {sleep }} \Delta t_{n}$ is constant and $P_{\text {sleep }}<P_{\text {on }}$, minimizing $E_{\Delta t, n}$ is equivalent to minimizing $t_{\mathrm{on}, n}$. Hence, one means to solve the problem expressed in (15) to (19) is to utilize a heuristic that minimizes the total time that small cells spend in the on state. This can be achieved by finding and switching on the best small cell for each user to connect to, so that the transmission of files from each small cell to users can be finished as quickly as possible to be able to put the small cells to sleep mode as soon as possible. Indeed, $t_{\mathrm{on}, n}$ can be expressed as a function of $t_{n, k}$, the time required for user $k$ to receive the data contained in the buffer of small cell $n$, for all users $k$ connected to small cell $n$. That is, $t_{n, k}$ can be expressed as:

$$
t_{n, k}=\frac{f_{k}}{B_{n, k} \omega_{n, k}}
$$

where $f_{k}$ is the amount of data user $k$ has to receive, in bit, and $B_{n, k}$ the bandwidth allocated on small cell $n$ to user $k$, in $\mathrm{Hz}$. The goal is then to find the best small cell $n$ for user $k$ to connect to so that $t_{n, k}$ is minimized. Two parameters influence the choice of optimal small cell $n$, namely $B_{n, k}$ and $\omega_{n, k}$. A close form expression for $B_{n, k}$ is difficult to obtain since it is dependent on the considered scheduler. However, as considered in [38], $\omega_{n, k}$ can be expressed as:

$$
\omega_{n, k}=\zeta_{b} \log \left(1+\zeta_{s}^{-1} \gamma_{n, k}\right)
$$

where $\gamma_{n, k}$ represents the SINR on the link between small cell $n$ and user $k$, and $\zeta_{b}>0$ and $\zeta_{s}>0$, respectively, represent the bandwidth efficiency and the SINR efficiency [37]. A good way to minimize $t_{n, k}$ is then to find the small cell $n$ providing the highest SINR to user $k$. We propose a heuristic algorithm to solve this particular problem. This algorithm is illustrated by a flow chart in Fig. 5 .

In the considered algorithm, the macro BS determines the best small cell for a user to connect to. The decision is performed using three metrics, namely the SINR values of the UE small cell links, the data rate requirement of the UE, and the current load (or amount of available resources) of each small cell, which can be obtained by information reporting from the small cells via their backhaul links. Using these three metrics, the macro BS builds a list of small cell candidates for the UE, by sorting them by UE-small cell link SINR values. Then, small cell which cannot serve the UE, i.e., small cells which cannot allocate enough resources to fulfil the UE's data rate requirement, are removed from this list. Small cells in sleep mode, however, are assumed to be able to always serve a user since all their resources are available. The final step in the algorithm checks whether at least one small cell candidate remains in the list. If there is, then the UE can start the connection procedure with the first small cell of the candidate list (i.e., the small cell with the best SINR value). Otherwise, it means that no small cell can serve the UE. In this case, the UE remains connected to the macro cell BS only.

This heuristic algorithm can be applied to various macro-assisted energy savings schemes, each taking a different approach to, e.g, obtain the UE-small cell link SINR values and to turn on small cells. The three macroassisted energy savings schemes considered in this paper are described in details in the following sections.

\subsection{Uplink (UL) signalling-based scheme}

In the UL signalling based scheme, a UE sends a wakeup signal to discover sleeping small cells. Upon detecting a wake-up signal, a sleeping small cell transitions from the sleep state to the on state and starts transmission of 


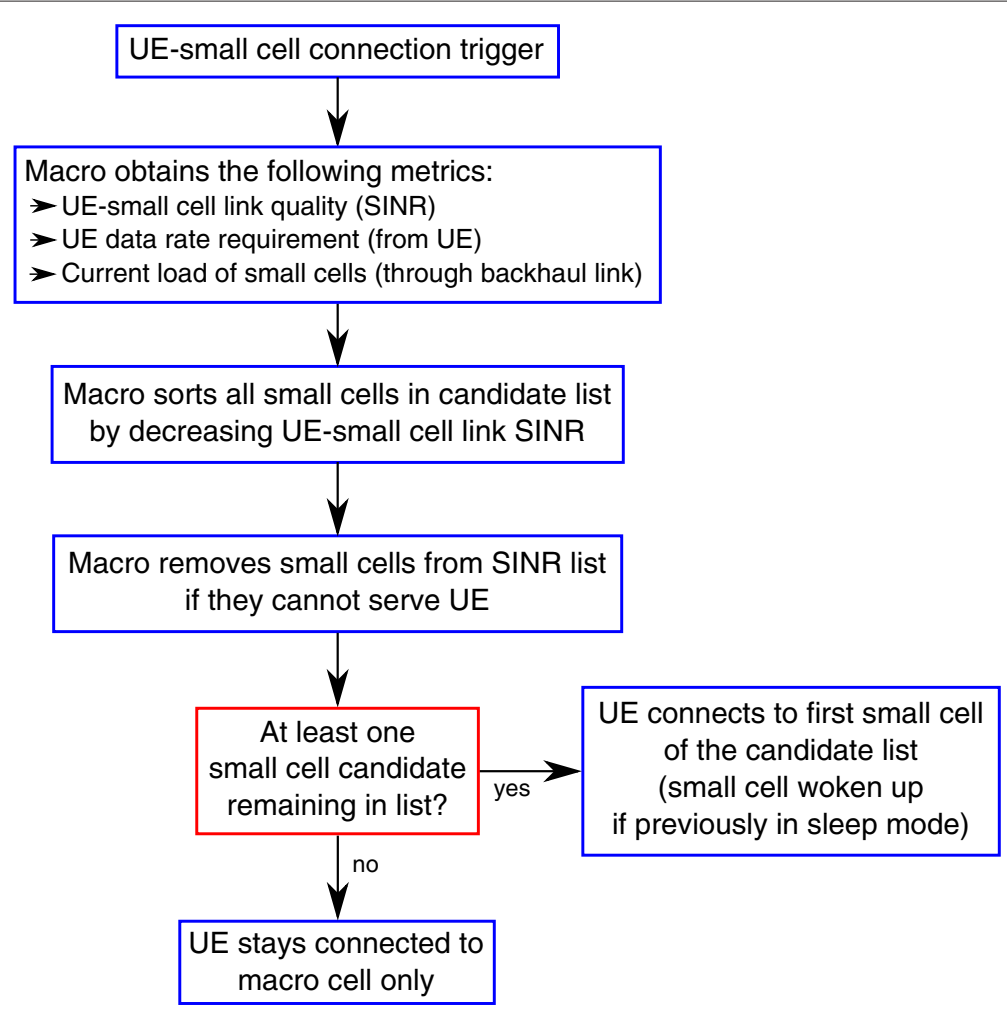

Fig. 5 Flow chart representing the heuristic algorithm used to determine the best small cell for a UE to connect to. The UE small cell link SINR can be obtained from varying sources depending on the energy savings scheme considered (see Sections 4.2 to 4.4)

pilot symbols which allow the UE to detect the small cell and perform the necessary measurements for connection establishment. For the small cell to be able to receive the wake-up signals from UEs, the receiving RF chain of the small cell needs be switched on even in sleep mode. The UL signalling-based scheme presented here is based on the UL-based small cell on/off scheme introduced in
[6], which is depicted in Fig. 6. The scheme in [6] does not consider dual connectivity capabilities for UEs and considers that UEs connect to small cells autonomously.

In this paper, we introduce a UL signalling-based energy savings scheme optimized for systems using the PCC architecture. A schematic representation of this macroassisted UL signalling based scheme is shown in Fig. 7.

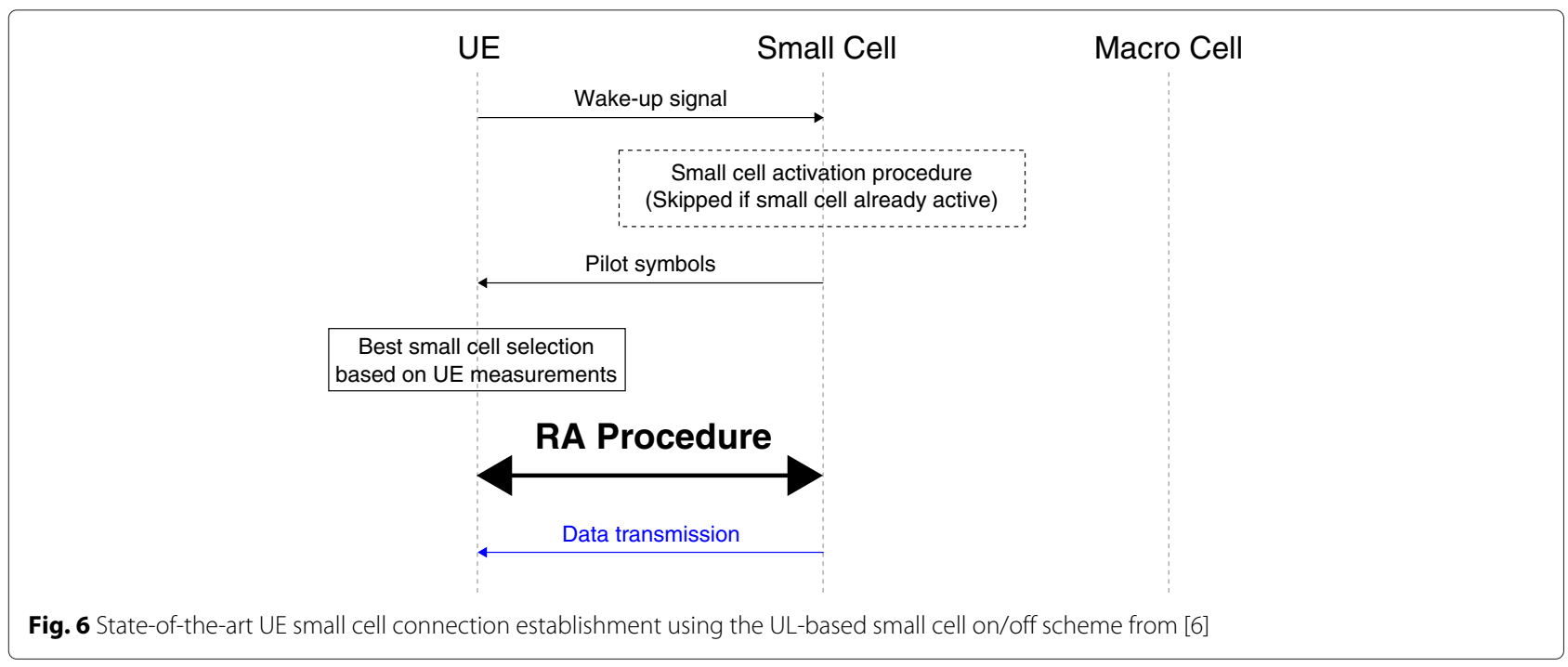




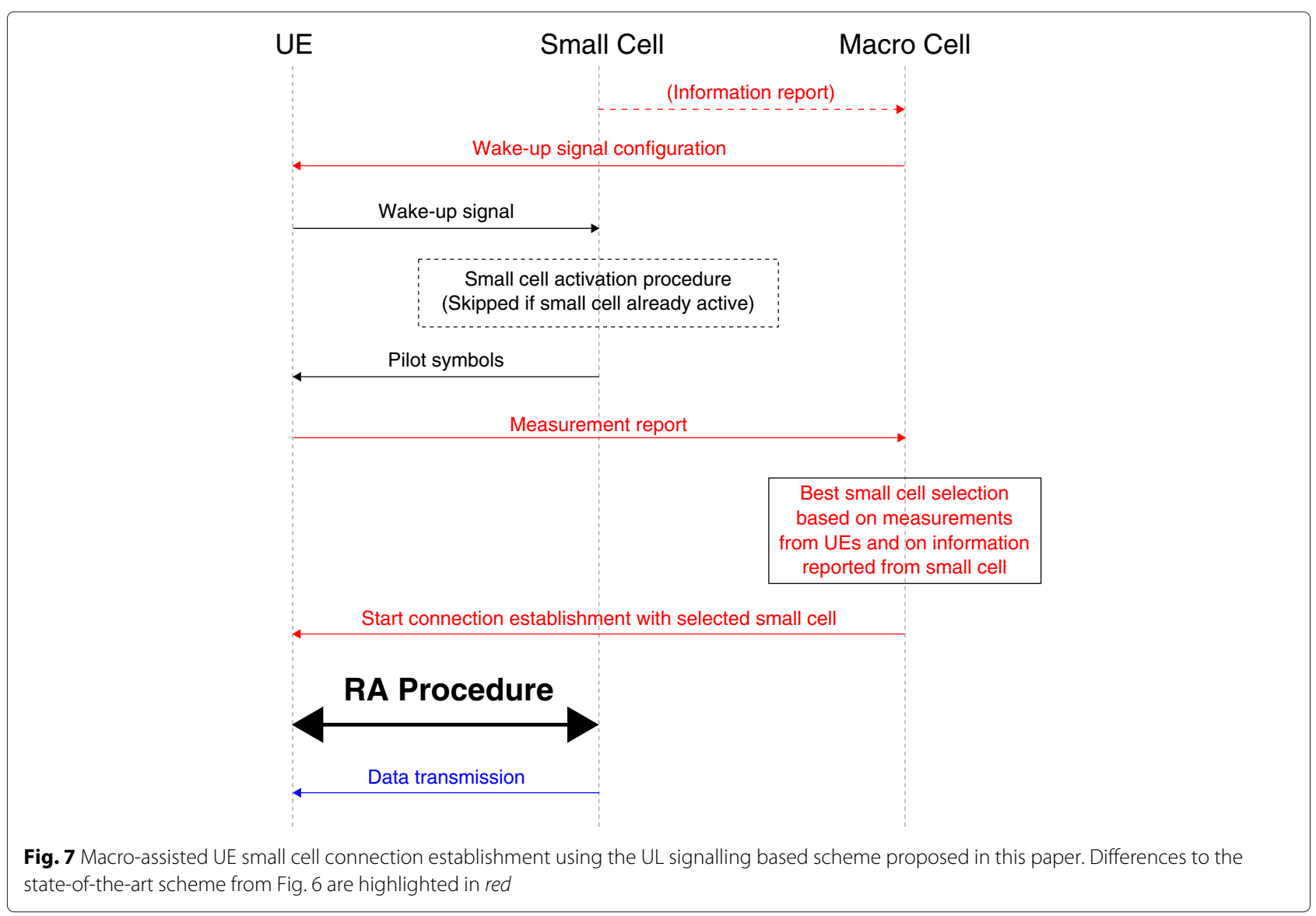

Differences from the state-of-the-art scheme of Fig. 6 are shown in red.

The UL signalling based scheme introduced in this paper has several advantages with respect to the stateof-the-art scheme from [6]. First of all, the macro cell can use the reported information from small cells in the on state, e.g., their current system load, during the connection establishment procedure, to help selecting the best small cell candidate for a UE. Furthermore, the fact that the macro cell is responsible for the UE small cell connection triggering event makes it possible for the macro cell to send a wake-up signal configuration message to the UE before the UE sends the wake-up signal, which can limit the effect of the wake-up signal so that it only affects a limited number of small cells, such as small cells belonging to a specific closed subscriber group (CSG), or small cells offering a specific amount of bandwidth.

\subsection{Downlink (DL) signalling based scheme}

The DL signalling based scheme takes its name from the fact that small cells in sleep mode still transmit pilot symbols to remain discoverable to users, albeit at a reduced rate compared to small cells in on mode. It is considered that sleep mode small cells transmit pilot symbols periodically, for a limited time during each period. For a small cell to be able to transmit these pilot symbols in the sleep mode, the RF transmitting chain and the power amplifier of the small cell need to be periodically turned on. Both the transmission of legacy CRS and new type DRS is considered for sleep mode small cell discovery in 3GPP $[25,31]$. In the case of PCC small cells, only the latter is applicable.

The DL signalling based scheme presented in this paper is based on the DL-based small cell on/off scheme introduced in [6], shown on Fig. 8. This state-of-the-art scheme considers that UEs autonomously connect to the small cell which offers the highest reference signal received power (RSRP). Furthermore, [6] does not specify how the small cells in sleep state should be turned on. This could be realized by the UE sending an activation signal to the small cell over the air interface, as depicted in Fig. 8, although this would require the RF receiving chain of the small cell to be turned on even in sleep mode. An alternative way, as proposed in [31], is to report the detection of the sleeping small cell to the cell currently serving the UE, which can in turn wake up the sleep mode small cell by sending a signal through a backhaul link. However, this implies that the UE is already being served by another cell, and that the 


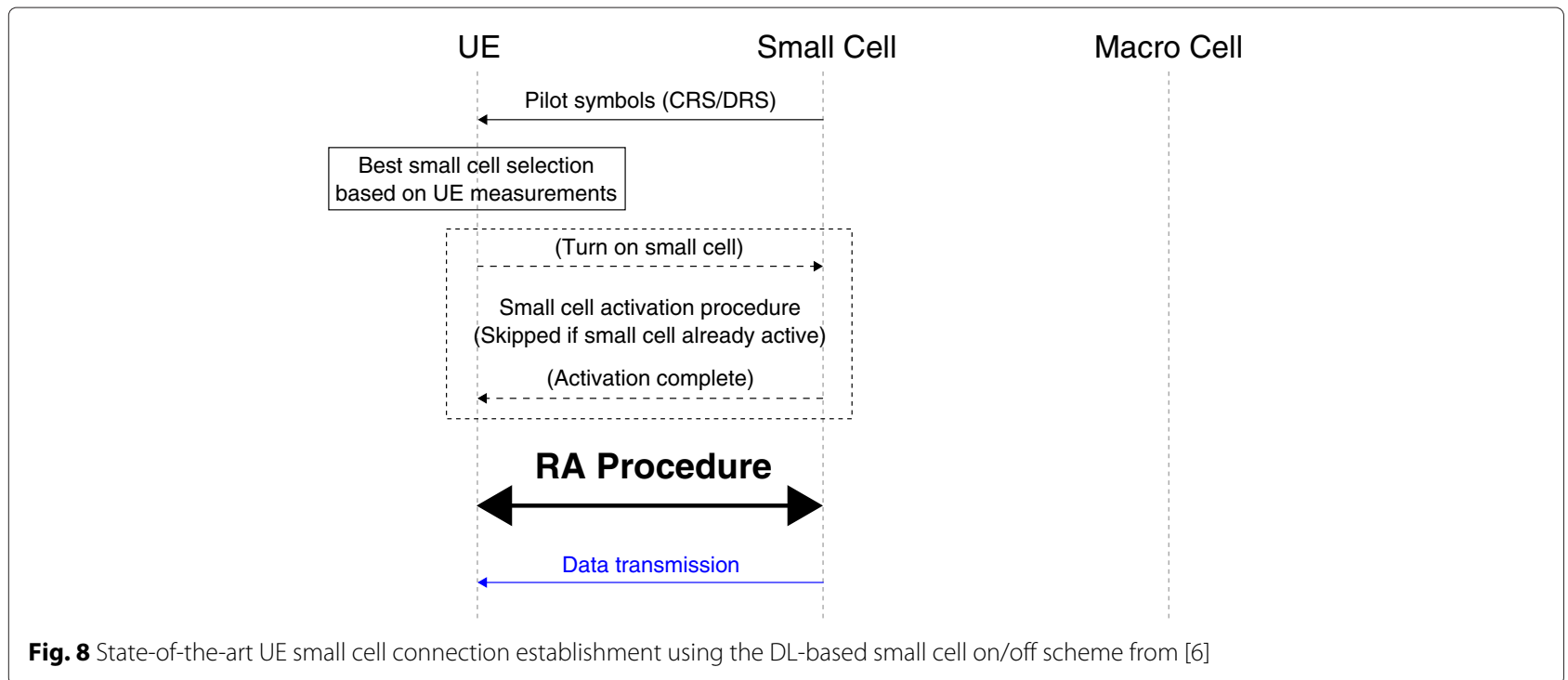

cell serving the UE has a direct backhaul connection to the desired target cell, which is not guaranteed in traditional HetNet deployments.

In this paper, we introduce a DL signalling based energy savings scheme optimized for the PCC architecture, implementing macro-assisted UE-small cell connection establishment. The schematic signalling flow of this scheme is shown in Fig. 9, where differences with respect to the state-of-the-art scheme from Fig. 8 are highlighted in red.

The DL signalling based scheme introduced in this paper not only makes it possible to use the additional reported information from the small cells to improve the best small cell selection, but also enables to solve the

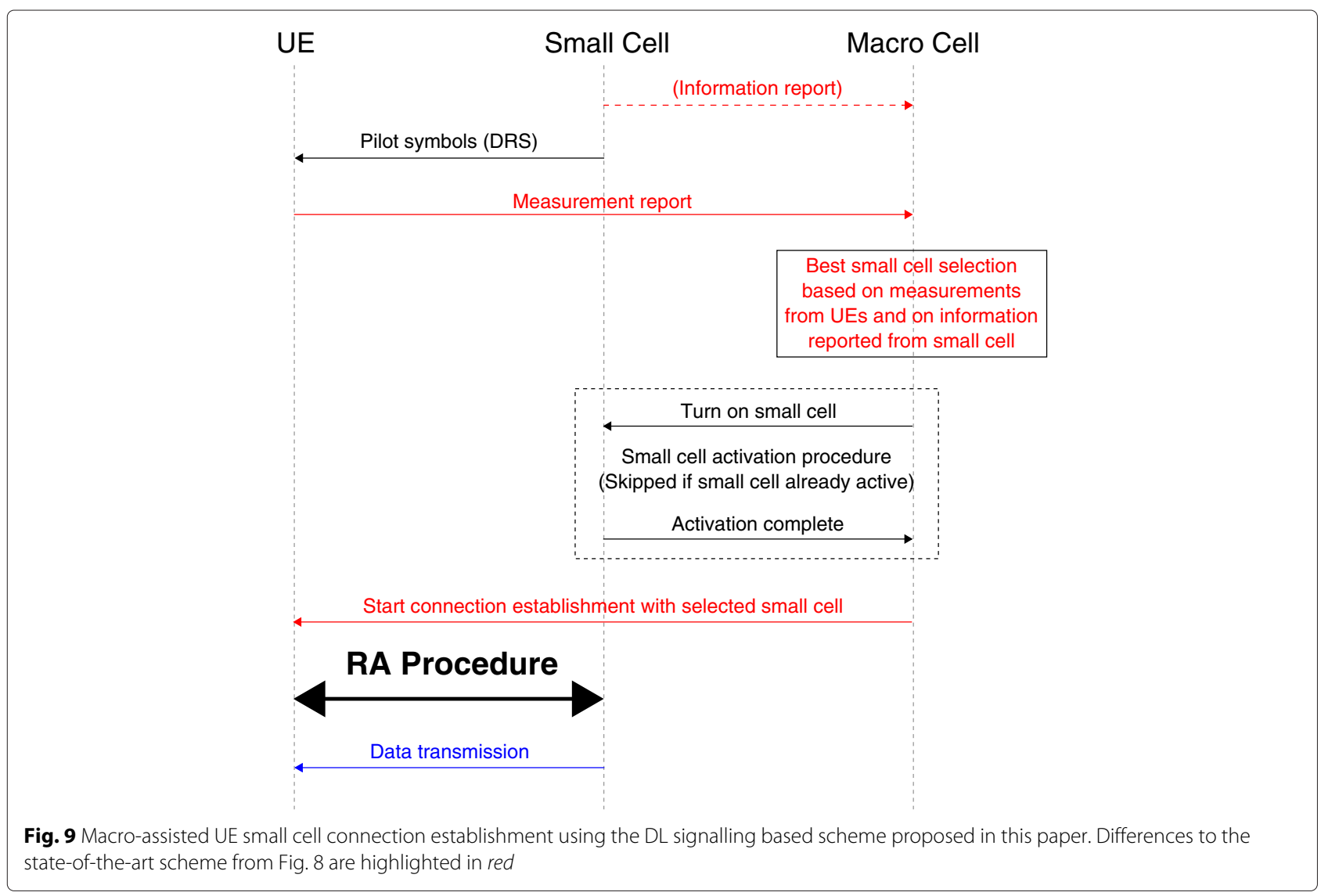


aforementioned issues regarding the activation of sleeping small cells. Indeed, since the PCC architecture considers that each small cell is connected to a macro cell by a backhaul link, small cells can always be woken up from the sleep state by the macro cell, instead of being woken up by the UE on the air interface, which guarantees that the RF receiving chain of a small cell can be switched off when this small cell is in sleep mode.

\subsection{Database-aided scheme}

The database-aided energy savings scheme is a macroassisted scheme in which a small cell in sleep mode neither transmits nor receives any signal on its air interface. This implies that UEs are neither able to discover small cells on their own, nor able to estimate the small cell channels of small cells in sleep mode. To compensate for this, each macro cell is equipped with a database, divided into $N_{S C}$ partitions, where $N_{S C}$ corresponds to the number of small cells attached to the macro cell, and where each partition of the database corresponds to one small cell. In each partition, past estimates of the UE small cell links are stored, mapped to sets of geographical coordinates $(x, y)$. This way, using the database and an estimate of a UE's position (e.g., reported from the UE), the macro cell can obtain the channel estimate required for UE small cell connectivity, even when small cells are in sleep mode [4, 5]. A drawback of the database-aided scheme with respect to the two other schemes is that a dedicated training phase is required to obtain a large enough number of past UE small cell channel links estimates before the scheme can be used. However, once the initial training is completed, the database can be updated during normal system operation by UE channel measurement reporting of active small cells configured by the macro cell. More details regarding the database training phase and update steps can be found in [4] and [5].

Notwithstanding, the database-aided scheme can potentially realize greater energy savings compared to the signalling based schemes because it does not require transmission or reception of any signals on the air interface during sleep mode.

Figure 10 depicts the UE-small cell connection establishment procedure using the database-aided scheme. The best small cell selection performed by the macro cell BS is based on three pieces of information obtained prior to starting the UE small cell connection procedure. These are the information reported from the small cells (e.g., their current load), the pilot symbol measurement reports of small cells in on state, and the reported geographical position information of the UE, necessary to obtain estimates of the UE small cell channels of the small cells in sleep state from the database. Small cells in sleep mode are woken up by the macro cell via the backhaul link, similarly to the DL signalling based scheme introduced in Section 4.3.

\section{Power model for small cells in sleep mode}

In this section, we determine the amount of energy consumed by a small cell in sleep mode, as a proportion of the amount of energy consumed by a small cell in on mode, for each considered energy savings scheme. In the context of small cell energy consumption, smaller BS sizes (pico cells and femto cells) are particularly relevant. However, in the PCC, small cells are operator-deployed [3]. Thus, the pico cell form factor is more appropriate than the femto cell form factor, mostly considered for userdeployed small cells. Therefore, we consider that small cells follow the power model used for pico cell BSs. Table 1 gives the energy consumption distribution in the various components of a pico cell BS according to the EARTH model described in [20].

As mentioned in Section 3.3, a small cell in the sleep state is required to transit to the on state in a very short time (e.g., [6] considers an activation time of $40 \mathrm{~ms}$ ), in order to guarantee the dynamicity required to implement the small cell energy savings schemes introduced in this paper. This short activation time can only be guaranteed if a number of components of the BS hardware, which take a non-negligible time to be switched on, stay on even when the small cell is in sleep mode [35]. The main power supply, consuming $P_{\text {main }}$, as well as the DC-DC power supply, consuming $P_{\mathrm{DC}-\mathrm{DC}}$, are required by small cells in sleep state for the most basic functions (e.g., wake-up signal reception through the backhaul link). Additionally, baseband components, consuming $P_{\mathrm{BB}}$, must stay on when the small cell is in the sleep state since they would require a very long time to be reactivated if turned off. The baseline power level, $P_{\text {baseline, }}$ representing the power consumed by small cell components which need to stay on even when the small cell is in sleep state, can therefore be computed as

$$
P_{\text {baseline }}=P_{\text {main }}+P_{\mathrm{DC}-\mathrm{DC}}+P_{\mathrm{BB}} .
$$

The value of $P_{\text {baseline }}$ is determined as $48 \%$ of the power level of a fully on small cell, according to the EARTH pico cell BSs model from Table 1.

The percentage of energy consumed by a small cell in sleep mode with respect to the energy consumed by a small cell in on mode varies depending on the considered energy savings scheme. The following subsections analyse this percentage for the three energy savings schemes introduced in this paper.

\subsection{UL signalling based scheme}

The UL signalling based energy savings scheme requires small cells in sleep mode to be able to receive wake-up signals from UEs, but they do not need to send any signal on their air interface. Therefore, only their RF-receiving chain is required to be left on in sleep mode (in addition to the baseline components), corresponding to half 


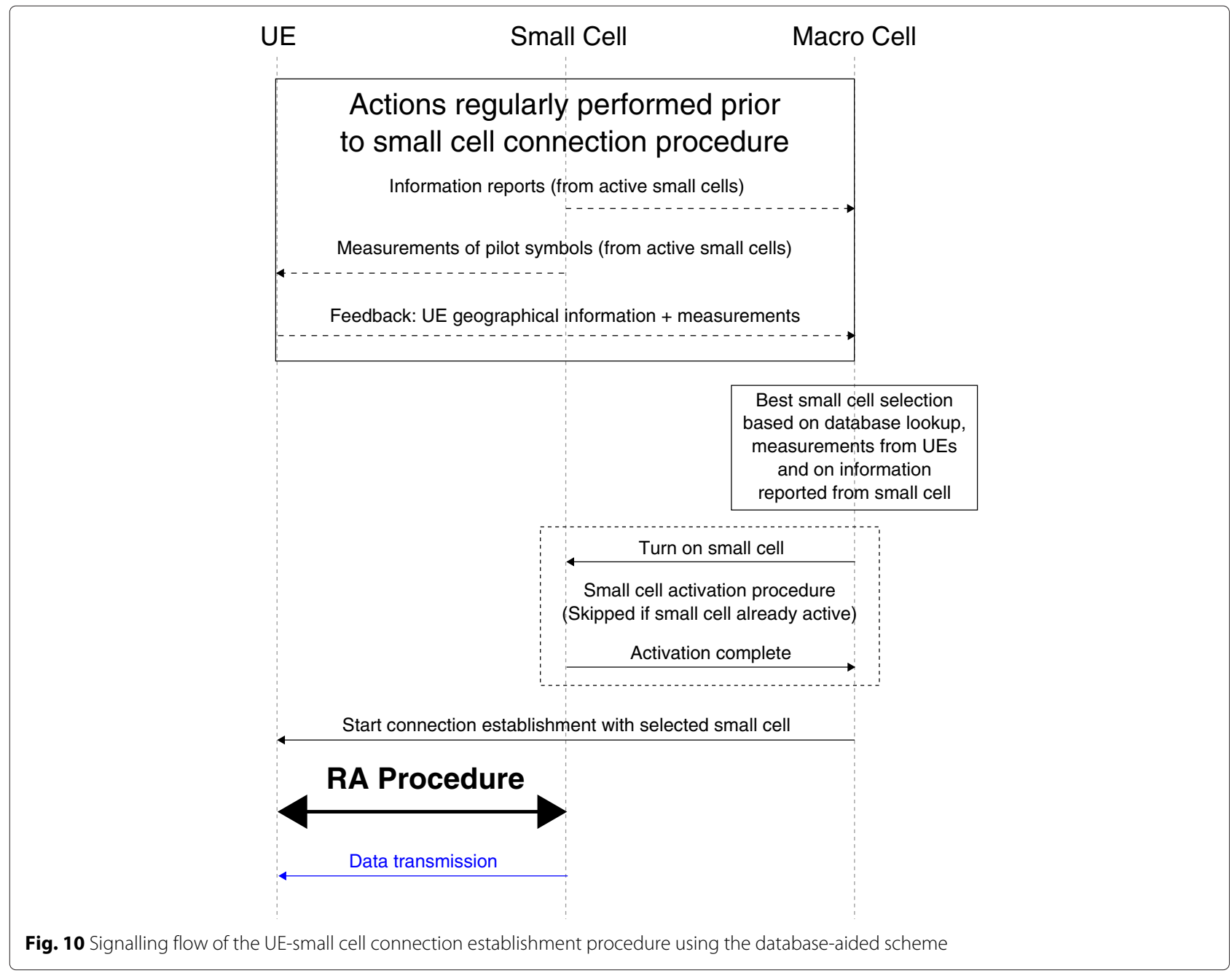

the power required for the whole RF chain. $P_{\mathrm{UL}}$, the sleep mode power consumption when the UL signalling based scheme is in use, is then computed as:

$$
P_{\mathrm{UL}}=P_{\text {baseline }}+\frac{P_{\mathrm{RF}}}{2} .
$$

$P_{\mathrm{UL}}$ is determined as $56 \%$ of the power level of a fully on small cell using values from Table 1.

Table 1 Percentage of the total energy consumption of pico cell BS components, according to the EARTH model [20]

\begin{tabular}{ll}
\hline BS components & Pico cell BS \\
\hline Baseband components & $33 \%$ \\
RF components & $16 \%$ \\
DC-DC power supply & $6 \%$ \\
Main power supply & $9 \%$ \\
Power amplifier & $36 \%$ \\
\hline
\end{tabular}

\subsection{DL signalling based scheme}

The DL signalling based energy savings scheme requires the small cell to be able to send discovery signals on its air interface in a periodic fashion. When the small cell sends these signals, the transmitting RF chain as well as the power amplifier of the small cell need to be on. However, when the small cell is not transmitting, these components are not required. $P_{\mathrm{DL}}$, the power consumed by a small cell in sleep mode when the DL signalling based scheme is in use, can therefore be expressed as:

$$
P_{\mathrm{DL}}=P_{\text {baseline }}+\left(P_{\mathrm{PA}}+\frac{P_{\mathrm{RF}}}{2}\right) \frac{\tau_{\text {transmission }}}{\tau_{\text {total }}},
$$

where $\tau_{\text {transmission }}$ corresponds to the time during which the small cell in sleep mode transmits discovery signals and $\tau_{\text {total }}$ corresponds to the total operation time. The ratio $\tau_{\text {transmission }} / \tau_{\text {total }}$ corresponds to the ratio between the pilot transmission duration and the pilot symbols transmission period $N / L$. For $N=10 \mathrm{~ms}$ and $L=200$ ms (see Section 6.3), $P_{\mathrm{DL}}$ is computed as $50 \%$ of the 
power level of a small cell in the on state using values from Table 1 .

\subsection{Database-aided scheme}

In the database-aided energy savings scheme, small cells are not required to interact in any way on their air interface. Therefore, the RF components and the power amplifier can be completely deactivated when the small cell is in sleep mode. This means that $P_{\mathrm{DB}}$, the power consumed by a small cell in sleep mode when the database-aided scheme is in use, is equal to the baseline power consumption $P_{\text {baseline, i.e., }} 48 \%$ of the power level of a fully on small cell.

A summary of all the percentages of power consumed by a small cell in sleep mode with respect to a fully on small cell for the various schemes is given in Table 2.

\section{Time required for UE small cell connection}

Small cell energy savings schemes provide energy savings but could also negatively impact user QoS and overall system performance. For instance, it takes a non-negligible amount of time for a small cell to transition from the sleep state to the on state. In addition, it takes a nonnegligible amount of time for the UE to establish a connection to a suitable small cell. All these delays could limit the availability of resources and subsequently impact the system performance. In this section, we quantify the time needed before a UE-small cell connection can be used to transfer data between the network and the UE for the different energy savings schemes. This gives some guidance on how long it takes for the resources at a sleeping small cells to be available for use. We follow this with an analysis of the impact of these delays on system performance in Section 7.

The interface and parameters for the UE small cell links considered in this analysis follow the state-of-the-art 3GPP assumptions [34]. Independently of the considered energy savings scheme, the connection procedure of a UE to a small cell is divided in two phases:

- The small cell selection procedure, which represents the connection steps until the macro cell has determined the best small cell for a UE to connect to. During this procedure, the UE can still receive data from the macro cell. The small cell selection

Table 2 Percentage of power consumed by a small cell in sleep mode with respect to a fully on small cell

\begin{tabular}{ll}
\hline Energy savings scheme & Percentage of power \\
\hline UL signalling based scheme & $56 \%$ \\
DL signalling based scheme & $50 \%$ \\
Database-aided scheme & $48 \%$ \\
\hline
\end{tabular}

procedure is different from one energy savings scheme to another.

- The RA procedure, which takes place after the UE has received a small cell connection establishment request from the macro cell, during which the UE actually connects to the selected small cell. Since the data to transmit needs to be transferred from the macro cell buffer to the small cell buffer during this procedure, data reception from the macro cell is unavailable during the whole RA procedure time. The RA procedure is independent of the small cell selection and therefore identical for all energy savings schemes.

In the next subsections, we describe the signalling flow and evaluate the connection establishment delays for the baseline macro-assisted connection setup with no energy saving features, the UL signalling based scheme, the DL signalling based scheme and the database-aided scheme.

\subsection{Baseline scheme}

In this section, we determine the required time to perform a UE small cell macro-assisted connection using a baseline scheme in which no energy savings features are considered. This means that a macro-assisted UE small cell procedure is employed, assuming that all small cells are always in the on state. Before the start of the UE small cell connection establishment, we consider that the UE is already connected to a macro cell BS. Furthermore, it is considered that the UE is already able to perform pilot symbol measurements from small cells in its vicinity to estimate the quality of the UE small cell channels and has already been feeding back these measurements to the macro BS.

As a consequence, whenever a small cell selection procedure is triggered, the macro cell can directly determine the best small cell candidate from the previously reported measurements and subsequently inform the UE of its decision. The delays to take into consideration in these operations are the processing delays in the BS, identified in Table 13.3 of [39] as $4 \mathrm{~ms}$, as well as the delay for the UE to receive and process the small cell connection establishment request message from the macro cell. Hence, we estimate that these two operations can be performed in a duration no longer than $10 \mathrm{~ms}$.

The UE subsequently uses the RA procedure to access and establish a connection to the selected small cell. During these procedures, a UE acquires full access to the small cell to be able to transmit and receive data through it. For this procedure, we assume a delay of $50 \mathrm{~ms}$. This delay can be calculated using Table 13.3 from [39], where the total idle-to-active delay is given to be

$$
47.5 \mathrm{~ms}+2 \times \mathrm{T}_{\mathrm{SC}-\mathrm{CN}}
$$


where $\mathrm{T}_{\mathrm{SC}-\mathrm{CN}}$ is the one-way propagation time of the signal over the backhaul link between the small cell and the $\mathrm{CN}$. Assuming a maximum delay of $50 \mathrm{~ms}$ means the maximum backhaul propagation time is $\mathrm{T}_{\mathrm{SC}-\mathrm{CN} \text {,max }}=$ $1.25 \mathrm{~ms}$ which, assuming backhaul signals propagate at the speed of light $\left(c=3 \cdot 10^{8} \mathrm{~m} \cdot \mathrm{s}^{-1}\right)$, allows a maximum backhaul length of $375 \mathrm{~km}$. This large distance provides flexibility for network operators to deploy RAN and $\mathrm{CN}$ nodes to meet different objectives.

After completion of the RA procedure, reception of data from the small cell buffer can start. An additional path switch operation may be required to update the serving gateway to send DL data for the UE directly to the connected small cell, but this operation has no influence on the data reception from the point of view of the UE. Therefore, only the $50 \mathrm{~ms}$ required by the RA procedure is taken into consideration.

The total time required for a UE-small cell connection procedure using the baseline scheme amounts therefore to $10+50=60 \mathrm{~ms}$.

\subsection{UL signalling based scheme}

In this section, we identify the required time to perform a UE small cell macro-assisted connection using the ULsignalling based energy savings scheme introduced in Section 4.2.

The detailed signalling flow for the small cell selection procedure when the UL signalling based energy savings scheme is in place is illustrated in Fig. 11. As explained in Section 4.2, the macro cell initially sends a wake-up signal configuration message to the UE in order to trigger the small cell selection procedure, and the UE sends a wake-up signal upon reception of the wake-up signal configuration message from the macro cell. The reception and detection of the wake-up signal by the small cells is not instantaneous but has a duration of $20 \mathrm{~ms}$ [6]. After the wake-up signal has been detected, a small cell needs enough time to turn on the components which were previously deactivated. This takes about $40 \mathrm{~ms}$ [6].

In PCC systems, the air interface of the small cells is required to be synchronized with the air interface of the

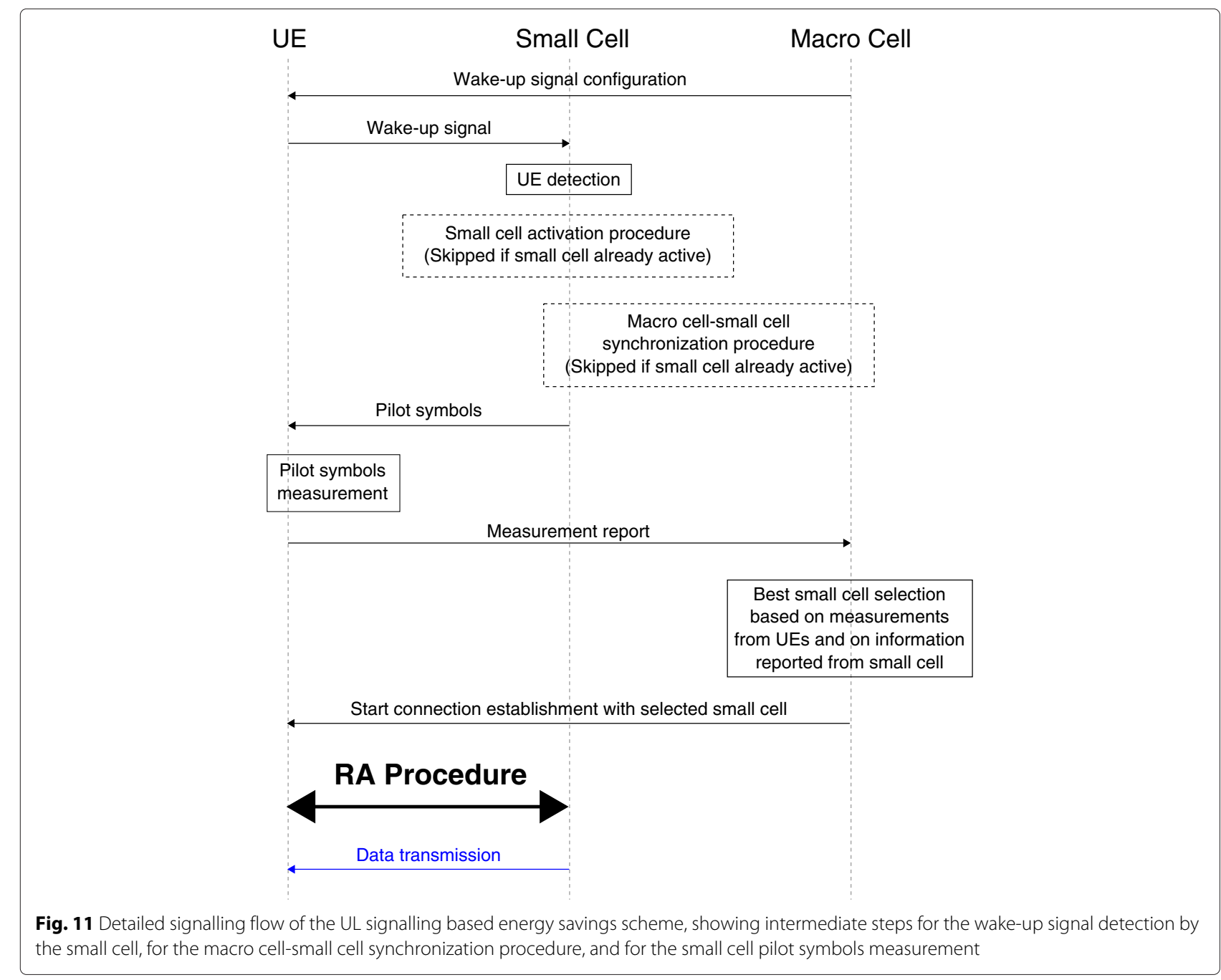


macro cell, so that UEs which are synchronized and connected to a macro cell are automatically synchronized to small cells [3]. In the baseline scheme, this synchronization has already been performed since UEs are able to receive and decode pilot symbols sent by small cells on their air interface. However, in the UL signalling based scheme, small cells woken up by a AU may need to synchronize their air interface with the macro cell prior to being accessible by UEs for channel estimation. This macro cell-small cell synchronization procedure can be performed in two ways:

- Through the macro cell air interface, by detecting synchronization signals broadcast by the macro cell on its air interface. This method does not require any information to be sent or received through the backhaul link, but requires the small cell to possess extra RF components capable of receiving signals from the macro cell air interface.

- Through the backhaul link, where the small cell sends a synchronization request to the macro cell, and receives the synchronization information back. This method has the advantage of not requiring extra RF components to access the macro cell air interface.

When the synchronization procedure is performed through the macro cell air interface, the small cell needs to detect the PSS and SSS of the macro cell it is connected to. To detect the first signal (PSS), the small cell performs matched filtering on each possible PSS pattern to determine the appropriate timing. The detection of the second signal (SSS) will determine the physical cell ID of the macro cell. This is necessary to identify the macro cell the small cell needs to synchronize to. Depending on the proximity of the small cell to the macro cell and on the channel conditions, these signals might not be detected with 100 $\%$ accuracy from the first attempt. However, in PCC systems, it is safe to assume that small cells are located in the vicinity of the macro cell they are connected to by backhaul link. Hence, a $100 \%$ detection accuracy assumption is realistic. Since PSS and SSS are both transmitted twice per radio frame $(10 \mathrm{~ms})$, and given the $100 \%$ detection rate, we can assume that detecting the PSS and the SSS can be done in a radio frame (i.e., $10 \mathrm{~ms}$ ). The total duration of the synchronization procedure is then $10 \mathrm{~ms}$.

If the synchronization procedure is performed through the backhaul link, the small cell first sends a synchronization request to the macro cell, the macro cell then processes the request and sends a message containing the synchronization information back to the small cell. After the small cell has processed the synchronization information from the macro cell, the synchronization procedure is complete. The time required to process the information at either the macro cell or at the small cell can be identified as $4 \mathrm{~ms}$ (see table 13.3 of [39]). The time required to send a message over the macro cell-small cell backhaul link is the one-way propagation time over this link $\mathrm{T}_{\mathrm{MC}-\mathrm{SC}}$. Requiring the synchronization procedure to be done in $10 \mathrm{~ms}$, to align with the over-the-air synchronization duration, requires this propagation time to be bound to $\mathrm{T}_{\mathrm{MC}-\mathrm{SC}, \max }=1 \mathrm{~ms}$. This allows a maximum macro cell-small cell backhaul length of $300 \mathrm{~km}$, which is much higher than the maximally considered distance between a macro cell and a small cell in PCC systems [3].

After synchronization, the physical DL channel is receivable and measurable. DRS-based measurements can be performed in order to estimate the channel. In the presence of fast-fading, more measurement samples are required in order to obtain accurate channel estimates [33]. A $200 \mathrm{~ms}$ measurement period provides a good compromise between measurement delay and channel estimation accuracy (see Section 5.5.3.2 of [40]). In practice, a shorter measurement duration can be used at the expense of potentially inaccurate measurements in relatively fastfading channel conditions.

Once the channel measurements are complete, the UE sends a report to the macro BS so that it performs the small cell selection. The macro BS requires about $10 \mathrm{~ms}$ to process the report, determine the appropriate small cell and send a connection establishment request message to the UE, as mentioned in Section 6.1.

Thus, when the UL signalling based energy-saving scheme is employed, the time required for the small cell selection procedure amounts to $280 \mathrm{~ms}(20 \mathrm{~ms}$ for the wake-up signal detection delay, $40 \mathrm{~ms}$ for the small cell activation delay, $10 \mathrm{~ms}$ for the synchronization delay, 200 $\mathrm{ms}$ for the DRS measurement delay, and $10 \mathrm{~ms}$ for the best small cell selection and UE notification). Taking into account the additional time of $50 \mathrm{~ms}$ required for the RA procedure, we obtain an overall time of $330 \mathrm{~ms}$ for the UE small cell connection to be fully usable for data transmission.

\subsection{DL signalling based scheme}

In this section, we identify the required time to perform a UE small cell macro-assisted connection using the DL signalling-based energy savings scheme introduced in Section 4.3. It is considered that the UE is able to receive pilot from all small cells, including small cells in sleep mode, before the start of the UE-small cell connection procedure. One of the particularities of this scheme is that the waiting time for periodic pilot symbols sent by sleep mode small cells to be received and measured by a UE, is not constant.

Figure 12 represents the periodic transmission of pilot symbols by the small cells. Two parameters influence the waiting time for pilot detection and measurements, namely the pilot symbols transmission period $(L)$, and 


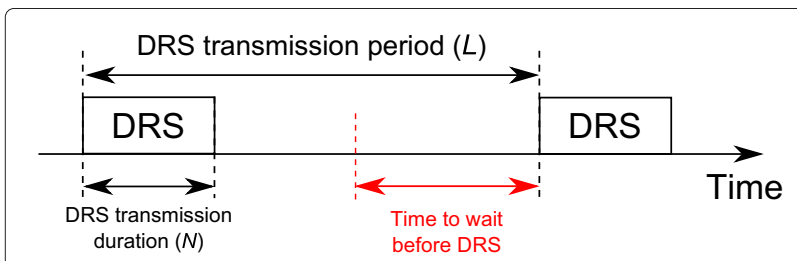

Fig. 12 Representation of the pilot symbols (DRS) transmission period $(L)$ and transmission time $(N)$, and of the waiting time before receiving the DRS

their transmission duration $(N)$. At any random instant, the pilot symbol detection time is $t_{\text {detect }} \in[0, L-N] \mathrm{ms}$. In the best case, there is zero wait time and in the worst case, there is $L-N$ wait time. If we assume a measurement duration of $N$ ms, this implies a delay of $t_{\text {measure }} \in[N, L]$ $\mathrm{ms}$ for a UE to detect and measure the pilot symbols.

For our considerations, a pilot symbol transmission period of $L=200 \mathrm{~ms}$ is considered (see [6]). Considering a very short (ca. $1 \mathrm{~ms}$ ) transmission duration $N$ allows the small cell RF transmitting chain to be deactivated most of the time, and hence to reduce the energy consumption of sleep mode small cells to a minimum. However, it has been suggested that channel measurements cannot be properly performed when such a short pilot symbol transmission duration is considered [31]. Transmitting pilot symbols for a longer period of time can increase the accuracy of channel quality measurements. A value of $N=10 \mathrm{~ms}$ is chosen, as this provides a good compromise between measurement delays, small cell energy consumption and measurement accuracy [33]. Thus, the time for a UE to detect and measure a small cell is bounded by [10 ms, 200 $\mathrm{ms}$.

Additionally, a macro BS requires $10 \mathrm{~ms}$ to process measurement reports, determine the best small cell and inform the UE. It also takes about $40 \mathrm{~ms}$ to activate the selected small cell. Therefore, the total time for the small cell selection procedure using the DL signalling-based energy savings scheme ranges between $60 \mathrm{~ms}$ and $250 \mathrm{~ms}$. Adding the $50 \mathrm{~ms}$ required for the RA procedure, the total time before actual data transmission can occur between the UE and a small cell with the DL signalling based energy savings scheme ranges from 110 to $300 \mathrm{~ms}$.

\subsection{Database-aided scheme}

In this section, we identify the required time to perform a UE-small cell macro-assisted connection using the database-aided energy savings scheme introduced in Section 4.4. One of the main advantages of this connection procedure is that the macro cell does not need to wait for any action from the UE before starting the small cell selection, as in the baseline scheme, since the data required for the small cell selection (i.e., the status information from small cells, the UE small cell channel estimations, and the UE's geographical position information) has either already been previously reported by UEs or is stored in the database. Nevertheless, the macro BS still requires about $10 \mathrm{~ms}$ to determine the best small cell and inform the UE. It also takes about $40 \mathrm{~ms}$ to fully activate the small cell. Therefore, considering the additional $50 \mathrm{~ms}$ required to perform the RA procedure, a total of $100 \mathrm{~ms}$ are required before the UE-small cell connection is usable for data transmission when the database-aided scheme is employed.

A summary of all the times required for the UE small cell connection procedure for each macro-assisted energy savings scheme is shown in Table 3.

\section{Performance results and analysis}

\subsection{Simulations model and parameters}

We use system-level simulations to evaluate the performance in terms of realizable energy savings and user QoS for the various energy savings schemes introduced in this paper. User QoS is measured in terms of average user throughput and average packet sojourn time. A total of five schemes are simulated:

- A baseline scheme not implementing energy savings where a UE small cell connection is established every time a UE starts receiving data. The connection is established to the small cell providing the highest SINR and able to match the UE's data rate requirement.

- The DL signalling based, UL signalling based, and database-aided energy savings schemes, in which a UE small cell connection is established every time a UE starts receiving data, using the heuristic algorithm shown in Fig. 5.

- A benchmarking scheme solving the energy consumption minimization problem expressed in (15) to (19), using the MM algorithm introduced in [36]. In this scheme, the algorithm is run (and hence the UE small cell connections changed) every time a user starts or finishes receiving data, so that the total energy consumption in the small cell network is always minimized. UE small cell connections are considered to be instantaneous and the proportion of power consumed by a small cell in sleep mode is

Table 3 Times required for UE small cell connection using the various macro-assisted schemes

\begin{tabular}{llll}
\hline Energy savings scheme & S.C. selection & RA & Total \\
\hline Baseline & $10 \mathrm{~ms}$ & $50 \mathrm{~ms}$ & $60 \mathrm{~ms}$ \\
UL signalling based & $280 \mathrm{~ms}$ & $50 \mathrm{~ms}$ & $330 \mathrm{~ms}$ \\
DL signalling based & $60-250 \mathrm{~ms}$ & $50 \mathrm{~ms}$ & $110-300 \mathrm{~ms}$ \\
Database-aided & $50 \mathrm{~ms}$ & $50 \mathrm{~ms}$ & $100 \mathrm{~ms}$ \\
\hline
\end{tabular}


chosen to be $48 \%$ of the power consumed by a fully on small cell, since we consider that only $P_{\text {baseline }}$ is consumed by sleep mode small cells in this scheme (see "Section 5" for details).

Regarding the database-aided scheme, two variants of databases are considered based on the type of UE small cell channel information stored [5]:

- Perfect database, where the database contains perfect estimates of instantaneous channel conditions between UEs and small cells. This represents an ideal scenario.

- Trained database, where approximate medium to long-term channel quality information between UEs and small cells are obtained through a training procedure [4] and may not represent instantaneous channel conditions.

The main simulation parameters are given in Table 4 . We consider a single site with three macro cell sectors with a cell radius of $290 \mathrm{~m}$. In each macro cell sector, a number of small cells and dual connectivity-capable UEs are dropped in a non-uniform clustered fashion, as considered in the 3GPP assumptions for small cell enhancements [34]. The number of small cells is fixed to 20, whereas the number of UEs is determined at the beginning of the simulation, varying from 10 UEs per macro cell, representing a low load situation, up to 60 UEs per macro cell, representing a high load situation. An additional number of legacy UEs are considered to be present in the network. Their number is unspecified, the only assumption being that they utilize $50 \%$ of the available resources in the macro cell.

The considered channel model is the ITU-R International Mobile Telecommunications (IMT)-Advanced multiple input multiple output (MIMO) channel model, widely used for system-level simulations involving small cells and HetNets [24, 34]. Depending on the radio link, different channel model scenarios are considered. For the UE macro cell channel, the ITU-R NLoS UMa scenario is chosen, and for the UE small cell channel, the ITU-R NLoS UMi scenario is selected.

Due to the different carrier frequencies considered for the macro cell and small cell links, no cross-tier interference is present in the system. The power transmitted by a small cells in on mode on its air interface is assumed to be $100 \%$ of the total small cell transmit power when the small cell is at least serving one user, and $5 \%$ of the total small cell transmit power when the small cell is not serving any user. This proportion can be explained by the fact that we consider that $5 \%$ of the radio frame is occupied by DRS pilot symbols (see section 3.1). Thus, small cells in the on mode not serving any user are potential sources of interference to other small cells. However, the power transmitted by small cells in the sleep mode is assumed to be negligible, even when the DL signalling based scheme is considered, due to the sparse periodicity of DRS transmission times in that case. As a consequence, sleep mode small cells do not generate any interference.

The simulation procedure consists in a DL-only data transmission on both the macro-UE and the small cell UE links. A non-full buffer (FTP) traffic model with an arrival rate $\lambda=0.5$ and a file size of $2 \mathrm{MB}$ is considered [41]. Each $\mathrm{UE}$ is randomly assigned a data rate requirement upon arrival of a new file addressed to it, drawn from a Rayleigh distribution with scale parameter $\sigma_{\text {req }}=1 \mathrm{Mbit} / \mathrm{s}$ [4]. The LTE physical time-frequency resource structure is assumed [42].

Resource allocation and scheduling procedures are performed independently in each macro cell and small cell $\mathrm{BS}$, according to a standard PF mechanism. The $50 \%$ of resources in the macro cell allocated to legacy UEs in the macro cell are not available to schedule UEs which support dual connectivity. The throughput on any given

Table 4 Main simulation parameters

\begin{tabular}{|c|c|c|c|}
\hline Parameters & Macro cell & Small cell & UE \\
\hline System bandwidth $(\mathrm{MHz})$ & 10 & 10 & - \\
\hline Carrier frequency $(\mathrm{GHz})$ & 2.0 & 3.5 & $2.0 / 3.5$ \\
\hline Total BS transmit power (dBm) & 46 & 37 & - \\
\hline $\begin{array}{l}\text { International Telecommunication } \\
\text { Union Radiocommunication Sector } \\
\text { (ITU-R) channel model scenario }\end{array}$ & Urban macro (UMa) non lineof-sight (NLoS) & Urban micro (UMi) line-ofsight (LoS) & - \\
\hline Duplexing scheme & FDD & TDD (mode 5) & FDD/TDD \\
\hline Number of antennas & 1 & 1 & 1 \\
\hline Antenna height (m) & 25 & 10 & 1.5 \\
\hline Antenna gain (dBi) & Max. 14 & 5 & 0 \\
\hline Receiver noise figure $(\mathrm{dB})$ & - & - & 9 \\
\hline Moving speed (km/h) & - & - & 3 \\
\hline
\end{tabular}


link is determined by a combination of the number of resource blocks (RBs) allocated, the channel quality, and the used modulation and coding scheme (MCS). A SINR to channel quality indicator (CQI) mapping is performed to determine the best MCS to use for a given channel quality.

While frequency division duplexing (FDD) is used for UE macro cell links, TDD is considered for the PCC UE small cell links [3]. UEs are expected to handle both FDD on the macro cell link and TDD on the small cell link. While this feature is technically challenging to perform with state-of-the-art equipment, discussions are under way in 3GPP to make joint FDD-TDD operation via carrier aggregation (CA) or dual connectivity technically possible [43].

Furthermore, it is assumed that UE stay connected to a small cell for a certain period of time after the last packet in their buffer has been transmitted, to avoid having to restart the whole small cell connection procedure if a new packet arrives shortly afterwards. This time before small cell disconnection is fixed to $50 \mathrm{~ms}$, a value large enough to minimize the probability of restarting the UE small cell connection procedure, but still smaller than the shortest UE small cell connection procedure time of $60 \mathrm{~ms}$ for the baseline scheme (see Table 3).

\subsection{Simulation results}

Performance results for three metrics are obtained, namely the achieved energy savings in the small cell networks, the average UE throughput and the average packet sojourn time, i.e., the average time which a packet spends in the network before it is fully transmitted. To compute the achieved energy savings, the state of each small cell (on state or sleep state) is recorded at each radio frame. Depending on its state, a small cell consumes either $100 \%$ of energy (for on state small cells) or a reduced percentage of energy (for sleep state small cells), as indicated in Table 2. Averaging these percentages over all the small cells and over the whole simulation time frame then yields the achievable energy savings quantity. The computation of the average UE throughput is performed by recording the size of each packet received by UEs within a radio frame, both from macro cells and small cells, divided by the duration of a frame $(10 \mathrm{~ms})$, and then by globally averaging these values. Finally, the average packet sojourn time is computed by recording the time each packet spends in the network before its complete transmission, then averaging all the recorded values.

Figure 13 shows the percentage of achieved energy savings by the various energy savings schemes. The MM algorithm outperforms all other schemes by at least 5 to $8 \%$, depending on the number of users in the network considered, offering a maximum of $52 \%$ of energy savings. The highest amount of energy savings achieved

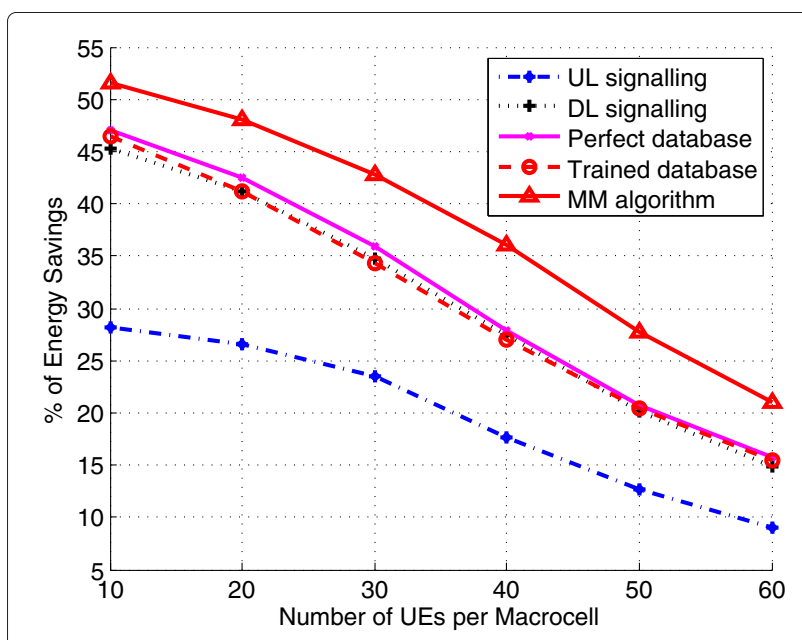

Fig. 13 Achieved energy savings by the various energy saving schemes

using the heuristic algorithm, reaching levels of above $45 \%$, can be obtained by the database-aided scheme when using the perfect database. Similar performance is obtained when using the trained database-aided scheme or the DL signalling based scheme. However, a strong performance degradation is observed for the UL signalling based scheme. This performance degradation is explained by the fact that all small cells in the vicinity of the UE are temporarily turned on after receiving the wake-up signal by setting on a timer, so that they become discoverable to UEs for at least the time of the connection procedure. The duration of this timer is selected to guarantee that enough time is provided to the small cell to synchronize to the macro cell, and to UEs to perform enough pilot symbol measurements to obtain a good estimation of the UEsmall cell channels. It is however possible to reduce the energy consumption of the UL signalling based scheme, and hence reduce this performance gap, by making use of the wake-up signal configuration message to limit the effect of the wake-up signal, and thus limit the number of small cells turned on during the connection procedure.

The energy savings presented in Fig. 13 only take the small cell network into consideration. The macro cell also consumes a significant proportion of the total energy consumed by the RAN. Assuming the 2012 state-of-the-art BS power consumption model used in the EARTH project [27], a 3-sector macro cell BS consumes $160.8 \mathrm{~W}$ of power, while a small cell (using the pico cell power model, as considered in Section 5) consumes $4.5 \mathrm{~W}$ of power, when all components of the BS circuitry are fully used. In our simulation model, 60 small cells are deployed per macro cell (20 small cells per macro cell sector, 3 sectors per macro cell), which means that small cells consume $63 \%$ of the total RAN energy. The maximum amount of energy savings achievable with the schemes introduced in this paper 
when considering the whole RAN is then around $30 \%$. However, this proportion can be increased if additional energy savings schemes, such as one of those discussed in Section 2, are used in the macro cell network as well.

Figure 14 shows the average UE throughput obtained with the various energy savings schemes using the heuristic algorithm, the baseline scheme not implementing energy savings, and the MM algorithm. First of all, we can observe that all energy savings schemes using the heuristic algorithm offer a performance improvement with respect to the baseline scheme, especially when a low number of users are present in the network. Up to $8 \%$ performance improvement can be obtained when using the trained database-aided scheme, while up to $25 \%$ performance improvement is achieved when using the UL signalling based, DL signalling based and perfect database-aided schemes. This performance improvement can be explained by the fact that less small cells are in the on state when using energy savings schemes, which reduces the global level of interference in the small cell network, thereby increasing the SINR of the UE small cell channels, enabling small cells to transmit data to UEs using a better MCS.

Additionally, we observe that that the performance of the UL signalling based, DL signalling based and perfect database-aided schemes are almost identical. Depending on the considered scheme, the UE is served by the macro cell for a short time (e.g., $50 \mathrm{~ms}$ for the database-aided scheme) or for a longer time (e.g., $280 \mathrm{~ms}$ for the UL signalling based scheme), as indicated on Table 3. Hence, the proportion of data which is received from the macro cell and from the small cell varies depending on the considered scheme. However, since the results show an almost identical performance, we can conclude that the QoS

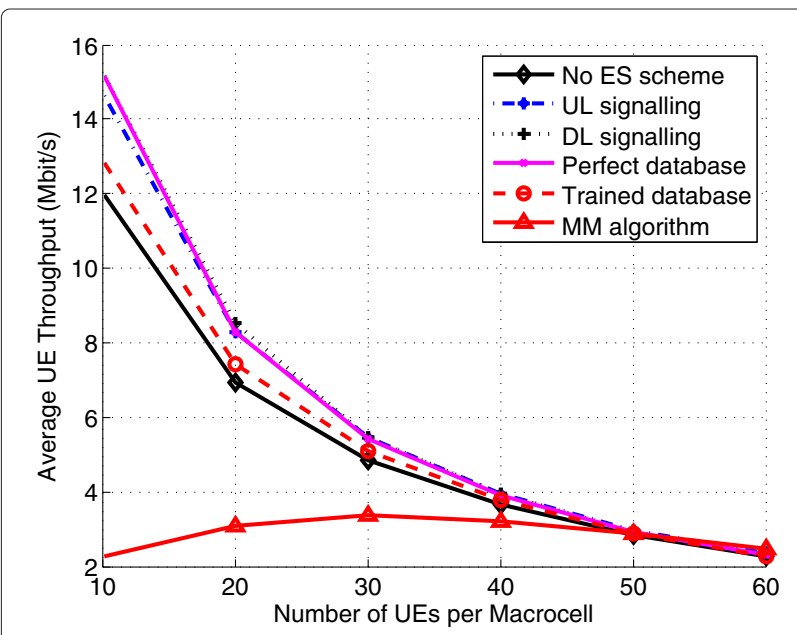

Fig. 14 Average UE throughput in Mbit/s. Results are displayed for the baseline scheme not implementing energy savings (ES), as well as for the various energy savings schemes obtained from the UEs when connected to the macro cell is similar to the QoS obtained when connected to a small cell. This can be explained by the fact that similar resources are available on the macro cell and on small cells, such as an identical bandwidth of $10 \mathrm{MHz}$, and that UEs can only receive data from a single point (either from the macro cell or from the small cell). However, when more bandwidth resources are available at the small cell compared to the macro cell, the different schemes show a marked performance difference (see [44]).

Furthermore, it is observed that the throughput performance when using the trained database does not degrade significantly compared to the performance obtained using a perfect database, and that the performance obtained with the trained database is still higher than the performance obtained with the baseline scheme. This implies that it is not necessary to strive to achieve perfect information, which may incur significant signalling costs to obtain, since the roughly trained database approaches the performance of the perfect database as the load in the network increases.

Finally, a relatively low throughput is obtained when using the $M M$ algorithm with respect to the other schemes. This can be explained by the fact that this scheme minimizes the energy consumption of the small cell network at all costs, while satisfying the minimum data rate requirements of users. The throughput performance is at its minimum when the number of users is very low, since in this case almost all small cells are in sleep mode (see Fig. 13) and most of the traffic is handled by the macro cell. In effect, the MM algorithm undertakes an aggressive version of the proposed heuristic where $t_{\mathrm{on}, n} \rightarrow 0$ for most small cells. As the number of users in the network increases, more small cells are turned on, which means that the traffic is handled by the small cell network in a higher proportion, where a higher fraction of bandwidth per user is available. The throughput then progressively decreases as the number of users increases, since each small cell then needs to serve a higher number of users on average.

Figure 15 shows the average packet sojourn time in millisecond. The observed behaviour of the packet sojourn time is highly correlated with the throughput results. For the schemes using the heuristic algorithm, the packet sojourn time steadily increases with the number of users in the network. When using one of the macroassisted energy savings schemes, the packet sojourn time is reduced when comparing to the baseline scheme not using energy savings since packets are transmitted faster, with a higher throughput. This sojourn time reduction is particularly observed for the perfect database-aided, UL signalling based and DL signalling based energy savings schemes, for which the throughput gains are the highest. However, the packet sojourn time is at its highest when the 


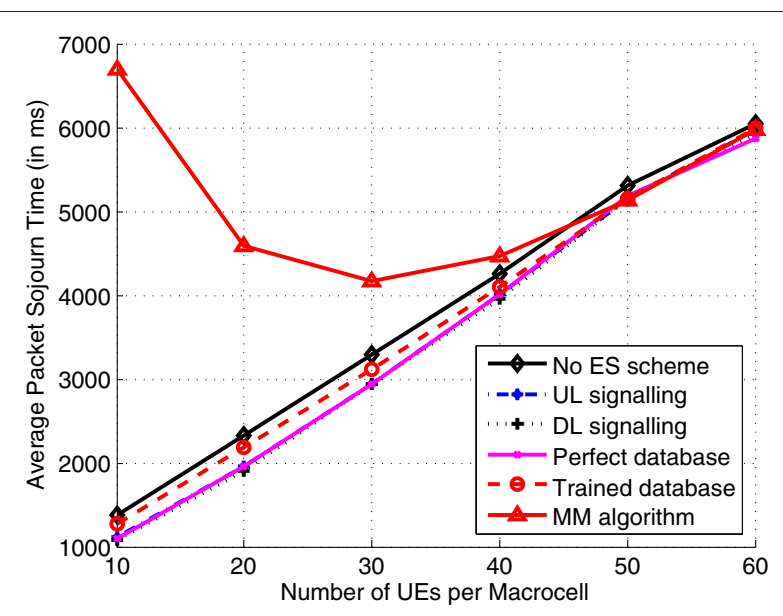

Fig. 15 Average packet sojourn time in millisecond. Results are displayed for the baseline scheme not implementing energy savings (ES), as well as for the various energy savings schemes

MM algorithm is used due to the relatively low achieved UE throughput in this case, except when a large number of UEs are present in the network.

Another question which needs to be asked is whether taking into consideration the signalling latencies during the UE small cell connection procedure has a negative impact on the system performance. To answer this question, we consider an additional benchmarking energy savings scheme, where it is assumed that macro-assisted UE small cell connections are instantaneous, as it is the case in $[4,5]$. The performance of this scheme with respect to the baseline scheme and to the perfect database-aided scheme is shown on Fig. 16. It can be observed that the throughput performance offered by the perfect database-aided

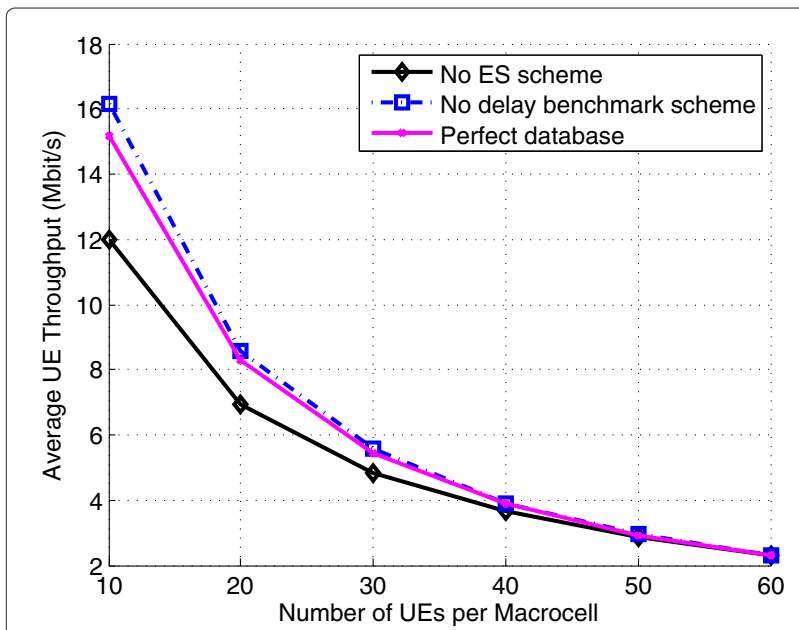

Fig. 16 Average UE throughput in Mbit/s. Results are displayed for the baseline scheme not implementing energy savings (ES), for the perfect database-aided scheme, and for a benchmark energy savings scheme where signalling latencies are ignored scheme and by the benchmarking scheme are almost identical. This can be explained by the fact that users receive a similar QoS from the network, regardless whether they receive data from a macro cell or from a small cell, since available resources on macro cells and small cells are comparable, as mentioned earlier in this section. The slight performance improvement brought by the nodelay benchmarking scheme with respect to the perfect database-aided scheme, in the case where a low number of users are present in the network, can be explained by the fact that the RA procedure, during which the delivered UE throughput is zero, is skipped in the benchmarking scheme. However, these conclusions are subject to change when the resources on the small cell are much higher than on the macro cell. The reader is invited to consult [44] for more information.

Even though our evaluations are based on DL data transmission, we expect the observed behaviour in the UL to be qualitatively similar. This is due to two factors. First, the use of TDD in the small cells implies that channel reciprocity can be assumed [42], and thus no additional resources are needed for UL channel estimation. Secondly, the UL is assumed to also use a reservation-based resource allocation mechanism. Thus, similarly to the DL, scheduling approaches that take energy savings criteria into account can also be employed.

\section{Conclusions}

In this paper, we have evaluated the performance of three energy savings schemes based on the concept of macroassisted UE small cell connection establishment, made possible by the PCC architecture, allowing small cells to be put to a sleep state when not needed. Two of these schemes, namely the DL signalling based energy savings scheme and the UL signalling based energy savings scheme, are an adaptation of state-of-the-art schemes to the PCC architecture, while the third scheme, the database-aided scheme, is natively enabled by the PCC architecture. The implementation of these schemes at low overhead for the network is made possible by a novel heuristic algorithm which connects a UE to a suitable small cell offering the highest SINR when a data connection is needed. Additionally, we have derived a power consumption model for a representative PCC small cell which allows to quantify the percentage of energy consumed by a small cell in the sleep state with respect to a small cell in the on state for the various energy savings schemes. Furthermore, we have determined the time required to perform a macro-assisted UE-small cell connection for each energy savings scheme, representing the delay before a UE can receive user data from a small cell.

System-level simulation results show that energy savings of more than $25 \%$ can be obtained using the UL signallingbased energy savings scheme, and energy savings of more 
than $45 \%$ can be reached when using the databaseaided or the DL signalling based energy savings scheme. Although the level of achieved energy savings is slightly lower than achievable energy savings when using state-ofthe-art optimization algorithms, the amount of signalling required to implement these macro-assisted techniques is much lower and makes them suitable for implementation in real network deployments. Furthermore, UE throughput performance improvement of up to $25 \%$ and a significant reduction of the average packet sojourn time can be observed when macro-assisted energy savings schemes are in use, in case a low number of users is present in the network, which is not the case when state-of-the-art energy consumption minimization techniques are in use. Finally, it has been shown that taking into consideration the latencies in the UE small cell connection procedures does not have a significant negative impact on the system performance. These conclusions can however be different if other assumptions are made regarding the system, e.g., if a significantly higher bandwidth is available on the small cells or if smaller file sizes are considered for data transmission [44]. These conclusions might also change if the capability of transmitting user data to UEs on both the macro cell and the small cell links is considered. In this case, a shorter connection time to the small cells may have a positive impact on the system performance, which means that the latencies present in the UE small cell connection procedure could play a bigger role.

In the future, we plan to further analyse the performance of the macro-assisted energy savings schemes introduced in this paper by allowing parameters, such as the load of the macro cell dedicated to legacy UEs, the time before small cell disconnection, or the bandwidth difference between small cell and macro cell, to vary.

\section{Competing interests}

The authors declare that they have no competing interests.

\section{Acknowledgements}

Part of this work has been performed in the framework of the FP7 project ICT-317669 METIS, which is partly funded by the European Union. The authors would like to acknowledge the contributions of their colleagues in METIS, although the views expressed are those of the authors and do not necessarily represent the project.

\section{Author details}

1 DOCOMO Communications Laboratories Europe GmbH, Landsberger Strasse 312,80687 Munich, Germany. ${ }^{2}$ University of Bremen, Dept. of Communications Engineering, Otto-Hahn-Allee NW1, 28359 Bremen, Germany.

Received: 31 October 2014 Accepted: 4 August 2015

Published online: 02 September 2015

\section{References}

1. N Bhushan, J Li, D Malladi, R Gilmore, D Brenner, A Damnjanovic, R Sukhavasi, C Patel, S Geirhofer, Network densification: the dominant theme for wireless evolution into 5G. Commun. Mag. IEEE. 52(2), 82-89 (2014)
2. PK Agyapong, M Iwamura, D Staehle, W Kiess, A Benjebbour, Design considerations for a $5 \mathrm{G}$ network architecture. IEEE Commun. Mag. 52(11), 65-75 (2014)

3. H Ishii, Y Kishiyama, H Takahashi, in Globecom Workshops (GC Wkshps), 2012 IEEE. A novel architecture for LTE-B :C-plane/U-plane split and Phantom Cell concept (Anaheim, CA, 2012), pp. 624-630

4. E Ternon, P Agyapong, L Hu, A Dekorsy, in IEEE WCNC'14 Track 3 (Mobile and Wireless Networks) (IEEE WCNC'14 Track 3: NET). Database-aided energy savings in next generation dual connectivity heterogeneous networks (Istanbul, 2014)

5. E Ternon, P Agyapong, L Hu, A Dekorsy, in The Eleventh International Symposium on Wireless Communication Systems 2014: Track 2: Networking, Protocols, Cognitive Radio, Wireless Sensor Networks, Services and Applications (ISWCS'2014 - Track 2-). Energy savings in heterogeneous networks with clustered small cell deployments (Barcelona, 2014)

6. 3rd Generation Partnership Project (3GPP), Technical specification group radio access network: 3GPP TSG RAN WG1 R1-133456, Views on Small Cell On/Off Mechanisms

7. EARTH Project Website. http://www.ict-earth.eu/

8. M Olsson, C Cavdar, P Frenger, S Tombaz, D Sabella, R Jantti, in Wireless and Mobile Computing, Networking and Communications (WiMob), 2013 IEEE 9th International Conference On. 5GrEEn: Towards green $5 \mathrm{G}$ mobile networks (Lyon, 2013), pp. 212-216

9. ETSI: Environmental Engineering (EE): The reduction of energy consumption in telecommunications equipment and related infrastructure. ETSI

10. EARTH Project Work Package 2: Deliverable D2.1: economical and ecological impact of ICT (2011). https://www.ict-earth.eu/publications/ deliverables/deliverables.html

11. W Li, H Zhang, W Zheng, T Su, X Wen, in Globecom Workshops (GC Wkshps), 2012. Energy-efficient power allocation with dual-utility in two-tier OFDMA femtocell networks (IEEE, Anaheim, CA, 2012), pp. 535-540

12. H Liu, W Zheng, $\mathrm{H}$ Zhang, Z Zhang, $X$ Wen, in Wireless Communications and Networking Conference (WCNC), 2013. An iterative two-step algorithm for energy efficient resource allocation in multi-cell OFDMA networks (IEEE Shanghai, 2013), pp. 608-613

13. P Frenger, P Moberg, J Malmodin, Y Jading, I Gódor, in Proceedings of the IEEE VTC 2011-Spring. Reducing energy consumption in LTE with cell DTX (Yokohama, 2011)

14. H Holtkamp, G Auer, H Haas, in Proceedings of the IEEE VTC 2011-Fall. On minimizing base station power consumption (San Francisco, CA, 2011)

15. A Ambrosy, O Blume, H Klessig, W Wajda. Personal Indoor and Mobile Radio Communications (PIMRC), 2011 IEEE 22nd International Symposium On (Toronto, ON, 2011), pp. 2418-2423

16. MJ Gonzalez, D Ferling, W Wajda, A Erdem, P Maugars, in Future Network Mobile Summit (FutureNetw), 2011. Concepts for energy efficient LTE transceiver systems in macro base stations (Warsaw, 2011), pp. 1-8

17. A Conte, A Feki, L Chiaraviglio, D Ciullo, M Meo, MA Marsan, Cell wilting and blossoming for energy efficiency. Wireless Commun. IEEE. 18(5), 50-57 (2011)

18. EARTH Project Work Package 4: Deliverable D4.1: Most promising tracks of green radio technologies (2012). https://www.ict-earth.eu/publications/ deliverables/deliverables.html

19. H Holtkamp, G Dietl, H Haas, in IEEE International Conference on Communications (ICC 2014). Distributed DTX Alignment with Memory, (2014). DOI:10.1109/ICC.2014.6883860

20. EARTH Project Work Package 4: Deliverable D4.3: Final report on green radio technologies (2012). https://www.ict-earth.eu/publications/ deliverables/deliverables.html

21. E Ternon, Z Bharucha, H Taoka, in Systems, Communication and Coding (SCC), Proceedings of 20139 th International ITG Conference On. A feasibility study for the detached cell concept (Deutschland, 2013), pp. 1-5

22. I Ashraf, F Boccardi, L Ho, in Personal, Indoor and Mobile Radio Communications Workshops (PIMRC Workshops), 2010 IEEE 21st International Symposium On. Power savings in small cell deployments via sleep mode techniques (Instanbul, 2010), pp. 307-311

23. I Ashraf, F Boccardi, L Ho, Sleep mode techniques for small cell deployments. Commun. Mag. IEEE. 49(8), 72-79 (2011)

24. 3rd Generation Partnership Project (3GPP), Technical specification group radio access network: 3GPP TR 36.842 V12.0.0, Study on Small Cell Enhancements for E-UTRA and E-UTRAN 
25. 3rd Generation Partnership Project (3GPP), Technical specification group radio access network: 3GPP TSG RAN WG1 R1-135514, Details of Small Cell On/Off with Small Cell Discovery

26. ETSI: Environmental Engineering (EE) measurement method for energy efficiency of wireless access network equipment. ETSI

27. EARTH Project Work Package 2: Deliverable D2.3: Energy efficiency analysis of the reference systems, Areas of Improvements and Target Breakdown, (2012). https://www.ict-earth.eu/publications/deliverables/deliverables.html

28. S Mohan, R Kapoor, B Mohanty, Latency in HSPA Data networks. Technical report, Qualcomm (2013)

29. 3rd Generation Partnership Project (3GPP), Technical specification group radio access network: 3GPP TSG RAN WG1 R1-131854, Network Adaptation for Small Cell Operation Efficiency Enhancement

30. Z Niu, J Zhang, X Guo, S Zhou, in Communication Systems (ICCS), 2012 IEEE International Conference On. On energy-delay tradeoff in base station sleep mode operation (Singapore, 2012), pp. 235-239

31. 3rd Generation Partnership Project (3GPP), Technical specification group radio access network: 3GPP TSG RAN WG1 R1-140191, Discussion on Discovery and Measurement for Small Cell On/off

32. 3rd Generation Partnership Project (3GPP), Technical specification group radio access network: 3GPP TSG RAN WG1 R1-140213, Discovery Signal Design for Small Cell On/off

33. 3rd Generation Partnership Project (3GPP), Technical specification group radio access network: 3GPP TSG RAN WG1 R1-140245, Performance Evaluation for Small Cell On/off Operation Considering Procedure and Discovery/measurement Enhancements

34. 3rd Generation Partnership Project; Technical specification group radio access network: 3GPP TR 36.932 V12.1.0, Scenarios and Requirements for Small Cell Enhancements for E-UTRA and E-UTRAN

35. LM Correia, D Zeller, O Blume, D Ferling, Y Jading, I Gódor, G Auer, L Van Der Perre, Challenges and enabling technologies for energy aware mobile radio networks. Commun. Mag. IEEE. 48(11), 66-72 (2010)

36. E Pollakis, RLG Cavalcante, S Stanczak, in Signal Processing Advances in Wireless Communications (SPAWC), 2012 IEEE 13th International Workshop On. Base station selection for energy efficient network operation with the majorization-minimization algorithm, (2012), pp. 219-223. arXiv:1202.1174v5

37. Z Ren, S Stanczak, P Fertl, F Penna, in Communications (ICC), 2014 IEEE International Conference On. Energy-aware activation of nomadic relays for performance enhancement in cellular networks (Sydney, NSW, 2014), pp. 2903-2908

38. K Majewski, M Koonert, in Telecommunications (AICT), 2010 Sixth Advanced International Conference On. Conservative cell load approximation for radio networks with shannon channels and its application to Ite network planning (Barcelona, 2010), pp. 219-225

39. 3rd Generation Partnership Project (3GPP), Technical specification group radio access network: 3GPP TR 25.912 V1 1.0.0, Feasibility Study for Evolved Universal Terrestrial Radio Access (UTRA) and Universal Terrestrial Radio Access Network (UTRAN)

40. 3rd Generation Partnership Project (3GPP), Technical specication group radio access network: 3GPP TS 36.331 V12.0.0, Radio Resource Control (RRC) Protocol Specification

41. 3rd Generation Partnership Project (3GPP), Technical specification group radio access network: 3GPP TR 36.814 V9.0.0, Further Advancements for E-UTRA Physical Layer Aspects (Release 9)

42. S Sesia, I Toufik, M Baker, LTE - The UMTS long term evolution: from theory to practice, 2nd edn. (John Wiley \& Sons Ltd, The Atrium, Southern Gate, Chichester, West Sussex, PO19 8SQ, United Kingdom, 2011)

43. 3rd Generation Partnership Project (3GPP), Technical specification group radio access network: 3GPP TR 36.847 V12.0.0, LTE Time Division Duplex (TDD) - Frequency Division Duplex (FDD) Joint Operation Including Carrier Aggregation (CA)

44. E Ternon, P Agyapong, A Dekorsy, Impact of varying traffic profile on Phantom Cell voncept energy savings schemes. Accepted for publication to VTC2015-Spring (2015)

\section{Submit your manuscript to a SpringerOpen ${ }^{\circ}$ journal and benefit from:}

- Convenient online submission

- Rigorous peer review

- Immediate publication on acceptance

- Open access: articles freely available online

- High visibility within the field

- Retaining the copyright to your article

Submit your next manuscript at $\boldsymbol{\nabla}$ springeropen.com 